\title{
Modelling Charge Transport for Organic Solar Cells within Marcus Theory
}

\author{
Riccardo Volpi
}

\section{hoU UNNEDNNG}


The cover is designed by Riccardo Volpi and licensed under the terms of the GNU Free Documentation License. The image of the trajectory of the CT state splitting is created by Sathish Kottravel of the visualization center in Norrköping. As can be noticed, the theory is always hidden in the back.

ISBN 978-91-7685-619-2

ISSN $0345-7524$

Printed by LiU-Tryck 2017 
To the present moment... 

With the technological advancement of modern society, electronic devices are getting progressively more integrated in our everyday lives. Their continuously growing presence is generating numerous concerns about costs, efficiency and the environmental impact of the electronic waste. In this context, organic electronics is finding its way through the market, allowing for potentially low-cost, light, flexible, transparent and environmentally friendly electronics. Despite the numerous successes of organic electronics, the functioning of several categories of organic devices still represents a technological challenge, due to problems like low efficiencies and stabilities (degradation over time).

Organic devices are composed by one or more organic materials depending on the particular application. The conformation and electronic structure of the organic molecules as well as their supramolecular arrangement in the single phase or at the interface are known to strongly affect the mobility and/or the efficiency of the device. While there is consensus on the fundamental physics of organic devices, we still lack a detailed comprehensive theory able to fully explain experimental data. In this thesis we focus on trying to expand our knowledge of charge transport in organic materials through theoretical modelling and simulation of organic electronic devices. While the methodology developed is generally valid for any organic device, we will particularly focus on the case represented by organic photovoltaics.

The morphology of the system is obtained by molecular dynamics simulations. Marcus theory is used to calculate the hopping rate of the charge carriers and subsequently study the possibility of free charge carriers production in an organic solar cell. The theory is then compared both with Kinetic Monte Carlo simulations and with experiments to identify the main pitfalls of the actual theory and ways to improve it. The Marcus rate between two molecules depends on the molecular orbital energies, the transfer integral between the two molecules and the reorganization energy. The orbital energies and the transfer integrals between two neighbouring molecules are obtained through quantum mechanical calculations in vacuum. Electrostatic effects of the environment are included through atomic 
charges and atomic polarizabilities, producing a correction both to the orbital energy and to the reorganization energy. We have studied several systems in the single phase (polyphenylene vinylene, $\mathrm{C}_{60}, \mathrm{PC}_{61} \mathrm{BM}$, triindoles) and at the interface between two organic materials (anthracene/ $\mathrm{C}_{60}, \mathrm{TQ1} / \mathrm{PC}_{71} \mathrm{BM}$ ).

We show how a combination of different methodologies can be used to obtain a realistic ab-initio model of organic devices taking into account environmental effects. This allows us to obtain qualitative agreement with experimental data of mobility in the single phase and to determine whether or not two materials are suitable to be used together in an organic solar cell. 


\section{Populärvetenskaplig sammanfattning}

I takt med samhällets teknologiska utveckling spelar elektroniska apparater en allt större och större roll i våra vardagsliv. Elektroniska apparaters kontinuerligt ökande förekomst skapar en rad olika frågeställningar kring deras kostnader och effektivitet, men även miljömässig oro kring elektroniskt avfall. Mot denna bakgrund har organisk elektronik seglat fram som ett alternativ till traditionell halvledarbaserad elektronik, med fördelaktiga egenskaper vad gäller materialens kostnad, vikt, genomskinlighet och miljöpåverkan. Trots att stora framsteg gjorts inom fältet är det fortfarande en avsevärd teknologisk utmaning att få organisk elektronik konkurrenskraftig i avseenden som effektivitet och stabilitet.

Organiska elektroniska komponenter består av ett eller flera organiska material, beroende på avsedd tillämpning. Såväl den geometriska formen och elektroniska strukturen hos de organiska materialen/molekylerna som materialens arrangemang i förhållande till varandra i en enda fas eller i ett gränsland mellan olika faser, spelar en avgörande roll för effektiviteten hos en komponent och dess förmåga att ombesörja laddningstransport. Även om förståelsen för den grundläggande fysiken bakom organiska komponenters funktion är god, är det ofta svårt att på teoretisk basis förklara vissa av deras egenskaper som avslöjas i experimentella studier. Denna avhandling fokuserar på att förbättra den teoretiska förståelsen för laddningstransport i organiska komponenter genom modelleringsstudier. Även om metodologin som utvecklas i avhandlingen är generellt tillämpbar, så fokuserar avhandlingen speciellt på organiska solceller.

De organiska materialens morfologi erhålls med molekyldynamiksimuleringar, medan s.k. Marcus-teori används för att beräkna hastigheten/sannolikheten med vilken en elektrisk laddning överförs från en molekyl till en annan. De erhållna resultaten jämförs både med resultat från s.k. Monte Carlo simuleringar och experiment, för att på så sätt identifiera såväl brister i teorin som strategier för att förbättra teorin. Sannolikheten för laddningsöverföring mellan två molekyler beror på molekylernas orbital-energier, på den s.k. överföringsintegralen mellan molekylerna, samt på molekylernas omorganisationsenergier. Orbital-energierna 
och överföringsintegralerna beräknas med kvantmekaniska metoder, medan omgivningens elektrostatiska effekter inkluderas genom atomära punktladdningar och polariserbarheter. I avhandlingen studeras ett flertal system i en enda fas (polyphenylene vinylene, $\mathrm{C}_{60}, \mathrm{PC}_{61} \mathrm{BM}$ ) och i gränslandet mellan två organiska material (anthracene/C60 TQ1/PC ${ }_{71} \mathrm{BM}$, triindoles).

Resultaten visar hur en väl vald kombination av olika metodologier kan användas för att erhålla en realistisk och tillförlitlig beskrivning av organiska solceller helt utan anpassning till experimentella data. Därmed har vi möjlighet att på teoretisk väg utforska huruvida två olika material är lämpliga att kombinera i organiska solceller. 
This thesis is the result of my PhD studies at Linköping University. A time span that lasted 4 years, full of joys and adversities in which I could experience and contribute to research in an international setting. This has been for me a period of great growth both at a professional and at a personal level, and I would like to take a moment to thank all the people that represented even a small piece of this wonderful experience.

My supervisor Mathieu Linares, for being such a passionate and active scientist. Thanks for the warm welcome and for introducing me to the $\mathrm{C} \pi \mathrm{C}$ "family", whose meetings I very much enjoyed every year, and hope to do the same in the future. Also thanks for the endless discussions and great fun we had in these years. Thanks to my co-supervisor Sven Stafström for the great research work culminating in this project, and for introducing me to it. This project has been financed by the Swedesh e-Science Research Center (SeRC) that is gratefully acknowledged also for promoting a modern innovative way of making Science. The heavy calculations that lead to the papers discussed in this thesis have been possible only thanks to the supercomputer resources and the technical support provided by the Swedish National Infrastructure for Computing (SNIC) at the National Supercomputer Center (NSC). In particular I would like to mention Johan Raber and Chandan Basu for the competent help. Thanks to all the colleagues and collaborators that contributed directly or indirectly to part of the work in this thesis. Prof. Ingrid Hotz and her student Sathish Kottravel from the Visualization Center in Norrköping for the project on the visualization of the KMC trajectories started in within SeRC. Maria Carmen Ruiz Delgado for the pleasant conversations at $\mathrm{C} \pi \mathrm{C}$ and for the collaboration on the Triindoles paper, that revealed to be a very interesting system to study. Prof. Demetrio Antonio da Silva Filho for the discussion in Linköping and for sending his student Leonardo Sousa in our group to start a collaboration on the $\mathrm{PC}_{61} \mathrm{BM}$ paper.

I would like to thank past and present colleagues of the Theoretical Chemistry (former Computational Physics) group, for all the interesting conversations, 
conferences and nice fika together! And of course the spring events. Thanks to Patrick Norman, Bo Durbeej, Mathieu Linares, Joanna, Thomas, Tobias, Dirk, Nanna, Paulo, Baswanth, Jun, Jonas S., Jonas B., Florent, Fang, Luiz, Olle, Cecilia, Morten and Racine. Thanks to Bo Durbeej, also for the engaging experience of being teaching assistant in your course, and thanks to Hassan Abdalla for sharing this enjoyable burden with me this year. Thanks to all the administration for being always helpful and available, in particular to Lejla Kronbäck for being a great administrative reference point for the group.

Thanks to the group of lunch (but not only), Arnaud, Tim, Teja and Hassan. Thanks to the many friends, met along the way, for making these years more interesting and fun. I won't venture here to list you all, but I am grateful for all the shared experiences and positive memories, as well as the negative ones.

Finally, a great thanks to my family, for the constant love and support, that is in no way altered by the distance. Grazie.

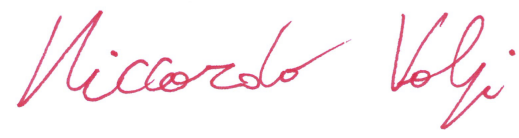

Riccardo Volpi

Linköping, December 2016 
1 Introduction 1

1.1 Organic Molecules and Polymers . . . . . . . . . . . . . . 2

1.2 Organic Solar Cells . . . . . . . . . . . . . . . . . . . . . . . . . . . . . . . . . .

1.3 Modelling Challenges . . . . . . . . . . . . . 6

2 Marcus Theory $\quad 9$

2.1 Marcus Formula . . . . . . . . . . . . . . . . . 9

2.2 CT state splitting diagram . . . . . . . . . . . . . . . 15

3 Marcus Parameters 21

3.1 Site Energies and Field Contribution . . . . . . . . . . . . . . 21

3.2 Transfer Integrals . . . . . . . . . . . . . . . . . . . . . . . . . 24

3.3 Reorganization Energies . . . . . . . . . . . . . . 26

4 Monte Carlo Simulations 31

4.1 The Kinetic Monte Carlo Algorithm . . . . . . . . . . . . . . . . . 32

5 Conclusions and Outlook $\quad 35$

5.1 Mobility in the Single Phase . . . . . . . . . . . . . . . . 35

5.2 CT state splitting at the Interface . . . . . . . . . . . . . . . 37

5.3 Future Perspectives . . . . . . . . . . . . . . . 37

Bibliography $\quad 39$

$\begin{array}{ll}\text { List of included Publications } & 49\end{array}$

Not included Publications

$\begin{array}{ll}\text { Paper I } & 55\end{array}$ 
$\begin{array}{ll}\text { Paper II } & 69\end{array}$

$\begin{array}{ll}\text { Paper III } & 95\end{array}$

$\begin{array}{ll}\text { Paper IV } & 109\end{array}$

$\begin{array}{ll}\text { Paper V } & 131\end{array}$

$\begin{array}{ll}\text { Paper VI } & 161\end{array}$

Appendices

A A Realistic Molecular Arrangement $\quad \mathbf{2 1 5}$

A.1 The Virial Theorem . . . . . . . . . . . . . . . . . 216

A.2 Force field energetic contributions . . . . . . . . . . . . . . . 217

A.3 Geometry Optimization . . . . . . . . . . . . . . . 218

A.4 Molecular Dynamics . . . . . . . . . . . . . . . . . . . . . 219

A.5 Canonical Ensembles . . . . . . . . . . . . . . . . . . 221

A.5.1 Berendsen temperature coupling . . . . . . . . . . 221

A.5.2 Nosé-Hoover temperature coupling . . . . . . . . . . . . . . 222

A.5.3 Berendsen pressure coupling . . . . . . . . . . . . . . 222

A.5.4 Parrinello-Rahman pressure coupling . . . . . . . . . . 223

A.6 The Ising Model . . . . . . . . . . . . . . . . . . . . . . . . 224

B Molecular Orbitals Theory $\quad 227$

B.1 Born-Oppenheimer Approximation . . . . . . . . . . . . . . . 227

B.2 Symmetrization and Antisymmetrization Operators . . . . . . . . . 229

B.3 Hartree-Fock . . . . . . . . . . . . . . . . . . . . . . 230

B.4 Semi empirical Methods . . . . . . . . . . . . . . 235

B.4.1 NDDO . . . . . . . . . . . . . . . . 235

B.4.2 INDO . . . . . . . . . . . . . . . . . . 235

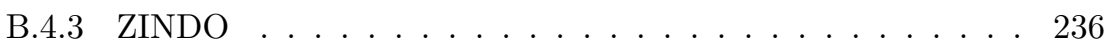

B.5 Density Functional Theory . . . . . . . . . . . . . . . 236

B.5.1 Kohn-Sham Scheme . . . . . . . . . . . . . . 238 


\section{CHAPTER 1}

\section{Introduction}

With the advancement of technology, electronic devices are getting progressively more integrated in our everyday life, with key components being transistors, LEDs and solar cells. Traditional electronic devices are mainly based on silicon or another inorganic material (Ge, GaAs, etc.). However, silicon is far from being an ecofriendly material [1] and a mass production of devices based on this material is not a sustainable long-term solution. Silicon production (in its pure and crystalline form) requires high amount of energy and potentially even environmentally harmful chemicals. Furthermore improper disposal of silicon devices at the end of their lifetime presents an environmental and health concern. In looking for alternative materials, nature represents a good source of inspiration. Carbon is a light abundant element, and with its four valence electrons is at the base of the complexity of all life-forms. Photosynthesis, as an example, has been slowly refined through billions of years till arriving to the efficient light-harvesting process that we are witnessing today. Also, carbon is belonging to the group IV of the periodic table, just on top of silicon, and it can be expected to have similar chemical properties.

Organic Electronics is a branch of Electronics based on organic materials, compounds containing carbon and hydrogen. Organic semiconductors are potentially light, flexible and transparent, allowing for the design of new futuristic devices [2-4]. Organic materials are also environmentally friendly and low cost to produce since they can be obtained by spin coating, ink-jet printing, lithography, etc. without the need of high temperatures or vacuum. Organic light-emitting diodes (OLEDs) and organic field effect transistors (OFETs) are already in commercial production and employed for thin displays and other simple devices. The performance of these organic components, "in some cases has already approached or even exceeded the requirements of the particular application" [2]. More importantly, cheap and low-power-consumption circuitry allows the integration of disposable and recyclable electronics in our everyday life [5], opening the door for innovative and revolutionary 
technologies.

One of the most important challenges of organic electronics is represented by organic solar cells, or organic photovoltaics (OPV). This is a problem of great importance also from an energetic point of view. In the search for an abundant and clean energy source, the Sun represents the ideal candidate. The total energy irradiated on Earth (5\% UV, $43 \%$ visible, $52 \%$ IR) exceeds the world's energy consumption by several thousands times [6]. The efficient conversion of such energy in a usable form represents the main technological challenge. This difficult task is attempted through solar cells, optoelectronic devices that transform sunlight into electricity. Conventional solar cells are built from inorganic materials, mainly based on silicon, and their efficiencies are approaching the theoretical ShockleyQueisser limit [7] for single junction solar cell [8-11]. Also, as a result of the high-energies required to obtain pure and crystalline silicon, the cost of inorganic solar cells is generally too high to allow their extensive and integrated use in daily life. Organic solar cells have reached a reported record efficiency of $\sim 11 \%[11,12]$. While this is still not enough to have a commercial interest, the competition with inorganic solar cells is not focused mainly on the efficiency, but more on the innovative applications originating from the particular properties of organic materials (flexibility, transparency, etc.). Besides the low efficiencies, another current problem of organic solar cells is their instability (degradation with time), due to factors like mechanical stress, humidity, oxidation, heat and even light absorption itself $[13,14]$. All these factors will cause structural modifications of the organic molecules composing the solar cell, with consequent modification of the supramolecular arrangement and decrease in performances and lifetimes of the devices. Many of these factors can be ruled out through particular techniques isolating the device, but it is of course not possible to isolate the solar cell from incident light. Photoisomerization is still one of the main challenging problems, in which structural changes between isomers is caused by photoexcitation. Thus great care must be taken in the choice of the organic materials to use and on how light absorption is affecting their supramolecular stacking.

\subsection{Organic Molecules and Polymers}

With the term organic materials we commonly refer to chemical compounds composed mainly by carbon and hydrogen. The semiconductive properties of these materials are principally determined by the four valence electrons of the carbon. The electronic configuration of a carbon atom in the ground state is $1 s^{2} 2 s^{2} 2 p^{2}$. The wavefunctions of the outer shell are close in energy and can mix in a linear combination to form hybridized orbitals. Hybridized orbitals on different atoms can then combine in $\sigma$ bonds (also called molecular orbitals) forming the backbone of the molecule, while the overlap of orthogonal atomic $p$ orbitals form a $\pi$ bond. Different hybridizations will lead to a different directionality of the $\sigma$ bonds and thus will affect the molecular geometry. $s p^{3}$ hybridization will lead to four $\sigma$ bonds in tetrahedral configuration. $s p^{2}$ hybridization will produce three $s p^{2}$ orbitals lying on a plane (oriented at a $\pi / 3$ angle) while the remaining $p$ orbitals are 
orthogonal to such plane. In $s p$ hybridization, two linear $s p$ orbitals are formed pointing in opposite directions and the other two $p$ orbitals remain unaltered. The semiconductive behaviour of conjugated polymers is mainly determined by the $\pi$ molecular orbitals. $[15,16] \sigma$ bonds are formed between the $s p^{2}$ orbitals of the carbon atoms composing the backbone of the polymer; from which the typical planar configuration of this class of materials. The remaining $p$ orbitals are orthogonal to the plane of the molecule and form the alternating $\pi$ bonds. These $\pi$-electrons result in spatially delocalized electrons. They belong to the whole $\pi$-segment and not to a specific carbon atom, becoming the main responsible for the conductivity of the molecule. A $\pi$-segment can be extended over the entire organic polymer or just over a part of it, depending on the specific polymer and its particular conformation. In general, all organic molecules present some similar behaviour, given by the presence of carbon chains in parts of their structure.

In the molecular ground state, the electrons fill the lowest energies orbitals with a maximum of two electrons of opposite spins each. In this way, for example for two carbon atoms, the two electrons that were in $p$ orbitals fill the $\pi$ level with opposite spins, while the $\pi^{*}$ orbitals remain empty. For a molecule, two important orbitals are the highest occupied molecular orbital (HOMO) and the lowest unoccupied molecular orbital (LUMO) (Figure 1.1). The ground state of
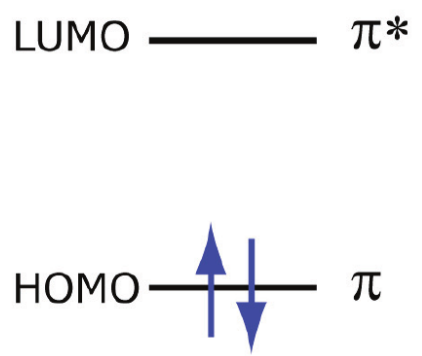

Figure 1.1. Highest occupied and lowest unoccupied molecular orbitals (HOMO and LUMO). Arrows denote two electrons with different spins.

the majority of organic molecules is electrically neutral and has total spin zero (is a singlet state). When one electron from the HOMO is raised to the LUMO, an exciton is formed on the molecule, usually localized on the $\pi$-segments of the molecule involved in the excitation. Electrons and holes are spin- $\frac{1}{2}$ particles, so the formed exciton can either be a singlet exciton or a triplet exciton.

\section{$1.2 \quad$ Organic Solar Cells}

Charge transport in organic materials is different in nature respect to the inorganic ones for two main reasons. First, excited electrons are not free to move as in inorganic crystals. An excited electron and its respective hole remain bounded together in an hydrogenoid system commonly called exciton. This is consequence of the poor screening of charges in organic materials due to low dielectric constants 
(typically $\epsilon_{r} \simeq 3.5$ ). Second, organic materials are "soft". A single charge carrier, hopping in an organic material, strongly interact with the vibrations on its nearby molecules, creating quantum quasiparticles called polarons.

In inorganic materials, when a photon is absorbed, an electron is passing from valence to conduction band and the electron and the hole formed in this process are free to move with respect to each other. In organic materials instead, due to the lower screening, the electron and the hole formed as a consequence of photon absorption are attracted to each other and form an hydrogenoid system called exciton. The dissociation of excitons into free charge carriers does not usually occur at room temperature, due to their strong binding energies. To overcome this problem, organic solar cells commonly utilize two different materials: an electron donor and an electron acceptor material. The exciton dissociation happens at the interface, where the electron likes to stay on the acceptor, while the hole likes to stay on the donor (Figure 1.2). This process generates a CT state [17-19]. There is

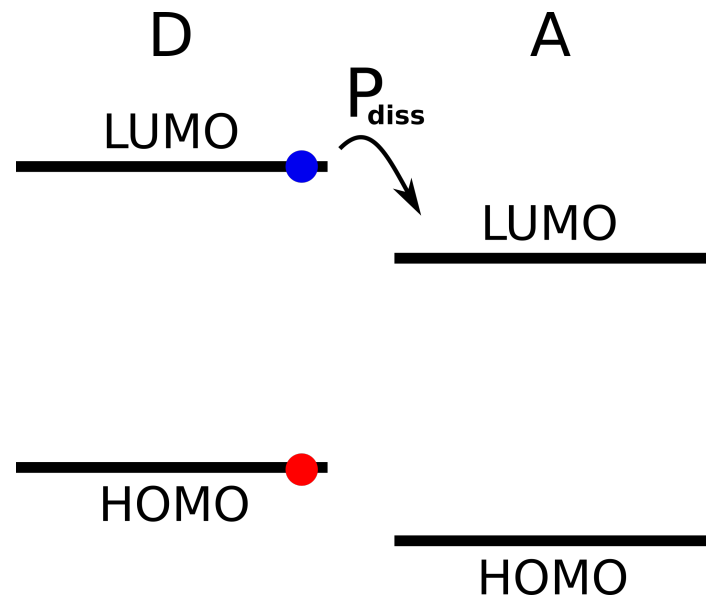

Figure 1.2. Schematic illustration of the exciton splitting mechanism at the interface between donor (D) and acceptor (A). The electron is represented in blue and the hole in red.

consensus in the literature on the main physical processes behind the functioning of an organic solar cell (Figure 1.3). [17,20-23] (1) A photon is absorbed with probability $\eta_{a b s}$, generating an exciton. (2) The exciton diffuses to the interface with probability $\eta_{e x},(3)$ dissociating in a charge transfer state (CT). (4) The CT state splits into free electron and hole with probability $\eta_{\text {diss }}$. (5) Now the charge carriers are free to move and can reach the electrodes with probability $\eta_{\text {trans }}$. Each one of these processes has some losses associated. (6) The exciton or ( 7 and 9) the CT state generated can recombine (radiatively) or (8) even free charge carriers can recombine in a CT state at the interface. Many of the processes here described are common to all organic devices; in particular in an OLED exactly the same processes are happening, just in reversed order.

In a blend of donor and acceptor material, it must be ensured that the excitons (created in either of these materials) can diffuse to the interface and thus allow the 


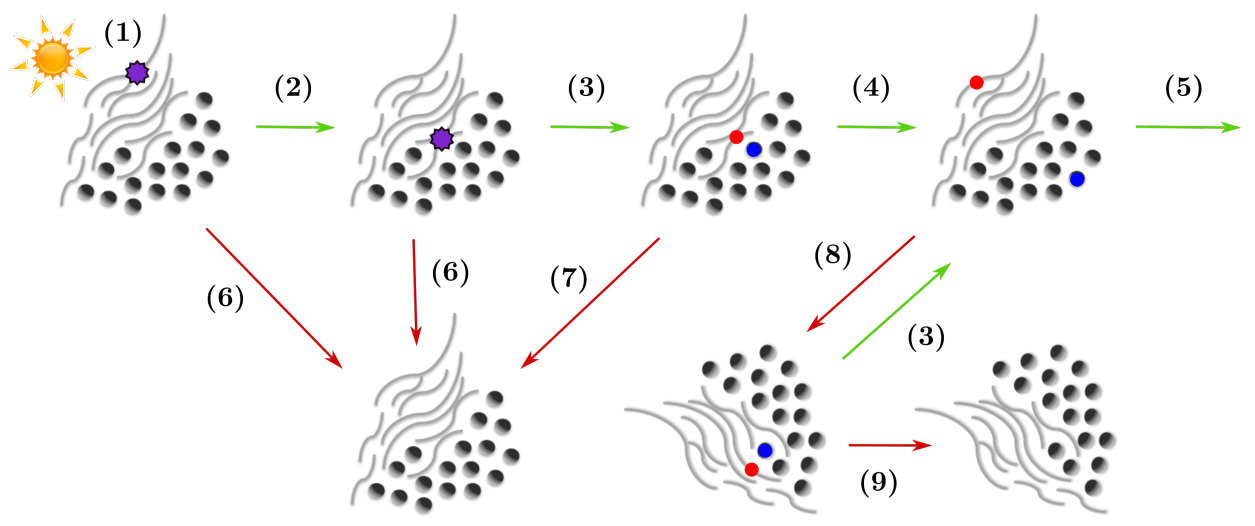

(1) light absorption and exciton formation

(2) exciton diffusion to the interface

(3) exciton dissociation into a CT state

(4) CT state splitting

(5) free charge carriers transport to the electrodes

(6) exciton decay

(7) CT state decay (geminate)

(8) free charge carriers recombination into a CT state

(9) CT state decay (non geminate)

Figure 1.3. Schematic illustration of an organic solar cell working principles. Figure adapted from [17].

charge separation process. Due to their short lifetime and low mobility, singlet excitons (usually the most abundant in an OPV) possess a diffusion length of about $10-20 \mathrm{~nm}$ [24]. Thus, anywhere in the active layer, the distance to the interface should be of the order of this average exciton diffusion length. On the other hand, a $20-40 \mathrm{~nm}$ double layer of donor and acceptor materials would let most incident photons pass freely through the device, without being absorbed. This imposes an important design condition on the interface morphology to enhance the charge generation, as illustrated in [25]. Depending on how the donor and acceptor are mixed together, it is possible to generate different types of junctions and interfaces. The most mentioned in the literature and easy to generate in the lab are the bilayer junction and the bulk heterojunction (BHJ). A bilayer junction contains two layers in between the conductive electrodes (flat interface). This interface favours the free charge carriers transport to the electrodes ( $\eta_{\text {trans }}$ in Figure 1.3), but only the excitons created in a thin layer around the interface will be able to reach the interface and thus dissociate (low $\eta_{e x}$ ). A bulk heterojunction (BHJ) consists of a three-dimensional nanoscale blend of donor and acceptor materials. In this interface the probability of diffusion to the interface $\eta_{e x}$ is very high, but it can result very difficult for the free charge carriers to find a path to the electrodes (low $\eta_{\text {trans }}$ ). Many other shapes and patterns are interesting efficiency-wise, as for example the interdigitated interface that represents a good compromise between 
exciton dissociation and free charge carrier transport [25]. Let us notice that in this simplified analysis we neglected the dependence of exciton dissociation and recombination on the interface pattern. Including these processes complicate significantly the picture and this case has to be studied more in detail. As a last comment, triplet excitons with a longer lifetime and consequently a longer diffusion length, are more likely to diffuse to the interface and split in free charge carriers. Being able to increase the population of triplet excitons in OPV would allow a boost in efficiencies. Research in this area is active through study of singlet-triplet fission [26] or Thermally Activated Delayed Fluorescence [27].

\subsection{Modelling Challenges}

After decades of research, the modelling of organic devices still remains nowadays a challenging task. The interesting properties of organic materials derives from a delicate intermix of physical phenomena. Organic materials are soft, every molecule is bounded to the neighbours through van der Walls forces. When an extra electron is added in these materials, significant deformations (and consequently changes in nuclear vibrations) are happening on the charged molecule and on the nearby ones. The electron will move in the material dragging around these deformations and thus creating an effective quasi-particle commonly called polaron. Supramolecular stacking, vibrations, excited states and polarization are all important aspects that need to be simultaneously considered to understand the dynamics of organic system. When dealing with crystalline materials, usually a Hamiltonian picture including phonons is employed. The Hamiltonians of the electrons and the lattice are interacting with each other and cannot be considered separately. This requires a coupled Hamiltonian (Holstein or Holstein Peierls) whose solution is non-trivial [2831]. The importance of the device morphology on the efficiency of an organic solar cell (Section 1.2) render very popular theoretical investigations based on simulations. Simulations employing drift-diffusion models $[25,32,33]$ evidence the significant contribution of morphology to the efficiency of solar cells on a global scale, $[25,33,34]$ but with a detail limited by the classical description employed. Since the efficiency of organic solar cells is greatly impacted by the processes that occur at the interface between the donor and acceptor a greater details is desirable. In amorphous materials the lack of crystallinity make the concept of phonons quite vague and invalid Bloch's theorem thus inhibiting charge delocalization. A model considering a charge localized on few molecules (but typically one) can be used and this charge is said to be hopping from one site to the next. Kinetic Monte-Carlo (KMC) simulations [35-45] have potentially the capability to model the quantum phenomena happening at the nanoscale, but the more details are included in the $\mathrm{KMC}$ scheme the more computational effort is required, limiting detailed KMC schemes to the study of interesting portion of a solar cell. The charge carriers hopping rate in $\mathrm{KMC}$ simulations can be calculated in several ways, the most predominantly used in the literature are the Miller-Abrahams rate and the Marcus rate. The Miller-Abrahams [46] formula is one of the simplest ways to couple temperature to the hopping rate. This is achieved by means of a Boltzmann factor 
helping to overcome the energy barrier for the transport. The Marcus hopping rate [47] can be derived from the Fermi golden rule (Chapter 2.1) and takes into account also the reorganization energy after each hop.

In this dissertation, we focus on charge transport in organic materials with particular attention to organic solar cells. We use a multiscale approach, using classical molecular dynamics (MD) simulations for the material morphology (10-50 $\mathrm{nm}$ ) and using computational chemistry methods to calculate the molecular orbitals on the single molecules $(\sim 1 \mathrm{~nm})$. To keep a flow for the reader both these sets of methods well known in the literatue, will be detailed in the appendices. A realistic morphology is used as a basis for charge transport considerations within Marcus theory (Chapter 2.1). This strategy works well to model the supramolecular stacking of organic materials in single phase and to create bilayer interfaces. As a further step we would like to create a deeper level of interpenetration as in a BHJ. One possibility is to start from a complete disorder mixture of molecules and try to simulate the evolution of the mixture till segregation with MD (Appendix A). This reveals to be a quite slow and computational expensive approach. An alternative way is to use Ising model to simulate this phase separation process $[36,48]$, subsequently fill the grid obtained with molecules and perform MD on the resulting system. This is the strategy explained in Appendix A.6. As already discussed in Section 1.2, the CT state splitting at the interface is one of the key processes determining the efficiency of an organic solar cell. The study of CT state splitting can be performed through Kinetic Monte Carlo simulations (Chapter 4), nevertheless it is not clear a priori for which range of electric fields the CT will split. An iterative process based on trial and error is needed to establish the meaningful set of electric fields, slowing down the whole analysis since a statistically significant ensemble of simulations are needed at each field value to test. To overcome this problem we will introduce the CT state splitting diagram (Section 2.2), an useful analytical tool allowing to study the possibility of CT state splitting and free charge carriers collection in function of the electric field. In particular, it is possible to determine if the chosen donor and acceptor material are suitable to be used together in an organic solar cell and if it is possible to find an electric field to favour simultaneously CT state splitting and free charge carriers collection at the electrodes. The parameters needed to compute the Marcus rates can be calculated as explained in Chapter 3 and all calculations regarding the electronic molecular orbitals are performed with well known computational chemistry methods like Hartree-Fock (HF) or density functional theory (DFT), briefly summarized for reader convenience in Appendix B. Once the parameters needed for the Marcus rates have been calculated and the interesting electric field range has been identified, the charge movement is simulated by means of a Kinetic Monte Carlo method, through a in-house program employing an atomistic description (Chapter 4 and papers). In a bottom-up fashion, considering progressively systems with increasing complexity, we started with studying charge carriers in the single phase and subsequently move to study several charge carriers and their interactions at the interface. In particular we then focus on the CT state splitting and the subsequent transport of free charge carriers to the electrodes. We will not consider in the present Dissertation the transport of excitons [49-51] or the coupling between the excited state, the CT state and 
the ground state [52-56] and we refer the reader to the appropriate literature for this. An overview of the results and aim of our research is presented in Chapter 5, followed by research articles. 


\section{CHAPTER 2}

\section{Marcus Theory}

As already introduced in Section 1.3 charge transport in organic materials reveals to be a challenging problem. One way of facing it, is to explicitly model the probabilistic nature of conduction in these materials, reducing the problem to finding reasonable approximations for the rates of the charge hopping from one molecule to another. A popular method widely employed in the literature is Marcus theory [47]. In this case the vibrational degrees of freedom of every molecule are treated classically. More sophisticated methods like Levi-Jortner theory [57-59] allow to consider quantized vibrations and vibrational tunnel effects, but they will not be treated in the present Dissertation. An interesting debate is present in the literature on how much these nuclear tunnel effects are important for conduction in organic materials [60], as discussed also in Paper V.

In the following chapter we will introduce Marcus Theory and in particular how this can be applied to study the interface of an organic solar cell.

\subsection{Marcus Formula}

Marcus Theory [47] was initially formulated to explain the rates of electron transfer reactions, i.e. those chemical reactions in which an electron moves from one chemical species (the electron donor) to another (the electron acceptor). It is widely used in the literature to calculate the charge carriers transfer rate between two molecules. Marcus Formula can be derived from Fermi golden rule and it expresses the rate of the hopping of an electron from the electronic ground state of the initial molecule $i$ to the electronic ground state of the final molecule $j$, moving always on the lowest of the vibrational energy curves.

In general terms, a quantum system is in a certain state $|\alpha\rangle$ and is perturbed with a time dependent perturbation $H e^{i \omega t}$ the transition probability per unit of 
time from a quantum state $|\alpha\rangle$ to another state $|\beta\rangle$ is given by the Fermi golden rule

$$
w_{\alpha \beta}=\frac{2 \pi}{\hbar}\left|H_{\beta \alpha}\right|^{2} \delta\left(E-E_{\beta \alpha}\right)
$$

where $E=\hbar \omega$ and we have assumed that $H$ is a "small" perturbation (in the sense of time-dependent perturbation theory). Let us consider a single molecule immersed in a continuous electric field $(\omega=0)$. According to Born-Oppenheimer approximation $|\alpha\rangle$ can be decomposed in an electronic part and a nuclear vibrational-rotational part, with quantum numbers $n_{\alpha}, v_{\alpha}$ and $r_{\alpha}$ respectively,

$$
|\alpha\rangle=|\psi\rangle \otimes|\phi\rangle=\left|\psi_{n_{\alpha}}\right\rangle \otimes\left|\phi_{v_{\alpha}}\right\rangle \otimes\left|\phi_{r_{\alpha}}\right\rangle \text {. }
$$

Since we are considering molecules in the solid state, we can imagine the rotational part to be a constant ground state. Every vibrational coordinate $q$ generates a manifold of vibrational states for $\left|\phi_{v_{\alpha}}\right\rangle$, usually approximated as states of an harmonic oscillator. The probability of an electronic transition from one electronic level $\left|\psi_{n_{\alpha}}\right\rangle$ to another one $\left|\psi_{n_{\beta}}\right\rangle$ can be calculated through the Fermi golden rule summed over all the possible vibrational transitions

$$
w_{n_{\alpha} n_{\beta}}=\sum_{v_{\alpha} v_{\beta}} w_{\alpha \beta}=\frac{2 \pi}{\hbar} \sum_{v_{\alpha} v_{\beta}} f\left(E_{v_{\alpha}}\right)\left|H_{\beta \alpha}\right|^{2} \delta\left(E_{\beta}-E_{\alpha}\right) .
$$

$f\left(E_{v_{\alpha}}\right)$ is the probability of being in the $\left|\phi_{v_{\alpha}}\right\rangle$ level given that the electronic level is $\left|\psi_{n_{\alpha}}\right\rangle$ and $H_{\beta \alpha}$ is the matrix element for the transition from $|\alpha\rangle$ to $|\beta\rangle$. Assuming to be in the "classical regime" for the occupation of the vibrational quantum levels, the probability is given by the Boltzmann factor

$$
f\left(E_{v_{\alpha}}\right)=\frac{e^{-\frac{E_{v_{\alpha}}}{k_{B} T}}}{\sum_{v_{\alpha^{\prime}}} e^{-\frac{E_{v_{\alpha}}}{k_{B} T}}} .
$$

As a further assumption, changing our vision of the harmonic oscillator from quantum to classical, we readjust the Boltzmann factor focusing now on the probability for the system of being in the position $q$. After normalization we can obtain the probability of being at $q$ as

$$
f(q)=\sqrt{\frac{\mu \omega}{2 \pi k_{B} T}} e^{-\frac{E(q)}{k_{B} T}},
$$

where $E(q)=\frac{1}{2} \mu \omega^{2}\left(q-q_{0}\right)^{2}$ is the classical energy for the position $q$ and $\mu$ is some sort of inertia associated with the coordinate $q$. According to Franck-Condon theory, the most probable transitions from the ground state of the harmonic oscillator are the vertical ones. In the charge transport context, we identify with $M=(i, m)$ a molecular orbital, composed by the index of a molecule $i$ and the quantum numbers $m$ of the orbital level on that particular molecule. Studying a hop from molecular orbital $M=(i, m)$ to molecular orbital $N=(j, n)$, we then identify $n_{\alpha}$ with $M$ and $n_{\beta}$ with $N$. The Fermi golden rule become

$$
w_{M N}=\frac{2 \pi}{\hbar} \int d q f(q)\left|H_{N M}(q)\right|^{2} \delta\left(E_{N}(q)-E_{M}(q)\right)
$$



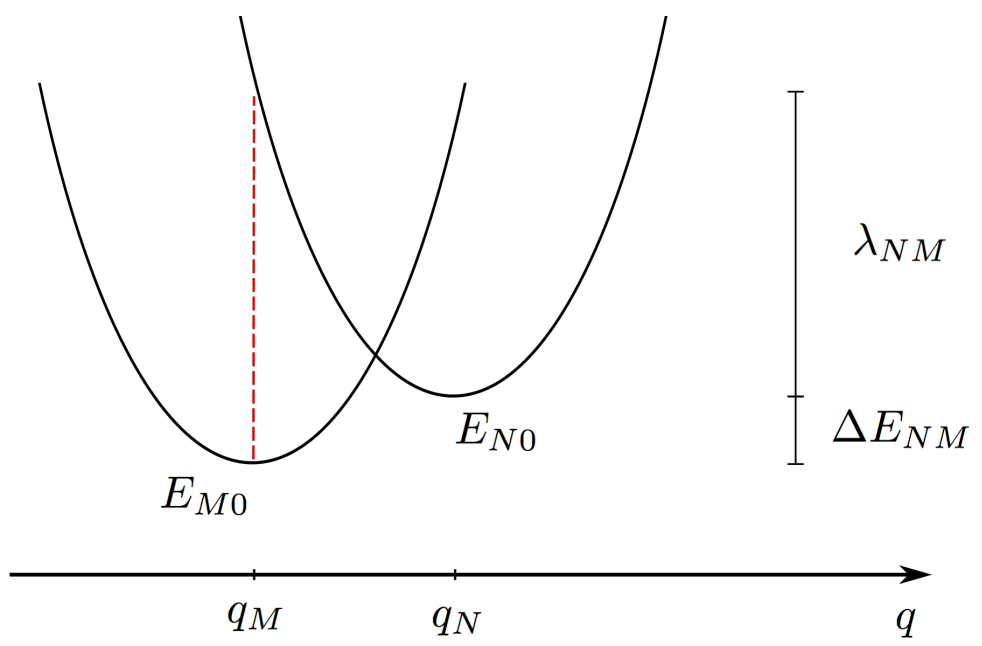

Figure 2.1. Illustration of the energetic potential in function of the vibrational degree of freedom $q$.

with $H_{N M}(q)$ the matrix element between the electronic levels $M$ and $N$ electron level assuming the value of the collective vibrational coordinate $q$ stays fixed (fixed nuclei). $H_{N M}(q)$ is commonly approximated by a single value $H_{N M}=H_{N M}\left(q_{e q}\right)$ and taken outside the integration sign. $q_{e q}$ can be the equilibrium value of the coordinate $q$ in the electronic level $M$ or some other kind of equilibrium value chosen for the problem at hand. Let us now develop the delta inside the integral (2.6). We know from distribution theory that

$$
\delta(f(x))=\sum_{i} \frac{\delta\left(x_{i}\right)}{\left|f^{\prime}\left(x_{i}\right)\right|}
$$

where the $x_{i}$ are the zeros of the function $f$. Approximating both the vibrational potential energy surfaces as parabolas with the same curvature, we obtain

$$
\begin{aligned}
E_{N}(q)-E_{M}(q) & =\frac{1}{2} \mu \omega^{2}\left(q-q_{N}\right)^{2}+E_{N 0}-\frac{1}{2} \mu \omega^{2}\left(q-q_{M}\right)^{2}-E_{M 0}= \\
& =\Delta E_{N M}+\frac{1}{2} \mu \omega^{2}\left(q_{N}^{2}-q_{M}^{2}\right)-\mu \omega^{2} q\left(q_{N}-q_{M}\right),
\end{aligned}
$$

where $E_{N 0}$ is the ground energy for the system with the extra charge in $N$ and $q_{N}$ is the equilibrium coordinate for the vibrational degree of freedom $q$ when the extra charge is on $N$. This is a linear function in $q$ and the zero of this function $q^{*}$ is the point in which the two energies (of initial and final state) are equal

$$
q^{*}=\frac{\Delta E_{N M}+\frac{1}{2} \mu \omega^{2}\left(q_{N}^{2}-q_{M}^{2}\right)}{\mu \omega^{2}\left(q_{N}-q_{M}\right)} .
$$

The delta of expression (2.6) becomes

$$
\delta\left(E_{N}(q)-E_{M}(q)\right)=\frac{\delta\left(q-q^{*}\right)}{\mu \omega^{2}\left|q_{N}-q_{M}\right|},
$$


that substituted in the (2.6) itself, gives

$$
w_{M N}=\frac{2 \pi}{\hbar}\left|H_{N M}\right|^{2} \sqrt{\frac{1}{4 \pi k_{B} T \lambda_{N M}}} e^{-\frac{\Delta E^{*}}{k_{B} T}} .
$$

$\Delta E^{*}$ is the classical barrier to overcome in passing from initial to final state and $\lambda_{N M}$ is the classical reorganization energy in the final state electronic configuration $N$ after a vertical excitation

$$
\begin{aligned}
\Delta E^{*} & =\frac{1}{2} \mu \omega^{2}\left(q^{*}-q_{M}\right)^{2} \\
\lambda_{N M} & =\frac{1}{2} \mu \omega^{2}\left(q_{M}-q_{N}\right)^{2} .
\end{aligned}
$$

Substituting the (2.9) into (2.12) and considering definition (2.13), we get, after several passages

$$
\Delta E^{*}=\frac{\left(\Delta E_{N M}+\lambda_{N M}\right)^{2}}{4 \lambda_{N M}} .
$$

and substituting this expression in (2.11) we obtain the Marcus transfer rate expression

$$
w_{M N}=\frac{2 \pi}{\hbar}\left|H_{N M}\right|^{2} \sqrt{\frac{1}{4 \pi k_{B} T \lambda_{N M}}} \exp \left(-\frac{\left(\Delta E_{N M}+\lambda_{N M}\right)^{2}}{4 \lambda_{N M} k_{B} T}\right) .
$$

We decide in our notation to explicitly write the contribution of the electric field on the hopping rate and thus for a hopping from molecular orbital $M=(i, m)$ to molecular orbital $N=(j, n)$ we will write

$$
w_{M N}=\frac{2 \pi}{\hbar}\left|H_{N M}\right|^{2} \sqrt{\frac{1}{4 \pi k_{B} T \lambda_{N M}}} \exp \left(-\frac{\left(q E \Delta z_{N M}+\Delta E_{N M}+\lambda_{N M}\right)^{2}}{4 \lambda_{N M} k_{B} T}\right),
$$

$\Delta z_{M N}$ is the z component of the $M \rightarrow N$ hopping movement and $q$ is the charge of the charge carrier performing this hop. Marcus formula eq. 2.16 involves the temperature $T$, the external electric field $E$ and three additional parameters depending on the system under consideration. These parameters have been presented during the derivation of Marcus formula made in this section and are namely: the transfer integral $H_{N M}$, the site energy difference $\Delta E_{N M}$ and the reorganization energy $\lambda_{N M}$. As detailed in Chapter 3 it is common practice in the current literature to calculate both the transfer integrals and the reorganization energies without any contribution from external field or extra charges. This approach is motivated by theoretical studies supporting the independence of transfer integrals and internal reorganization energy from the external electric field. [61,62] Furthermore the external reorganization energy (eq. 3.17) is independent of the external field and additional charges, due to the linear polarization method used, as demonstrated in [63]. The only parameter influenced by the field is thus the energy difference $\Delta E$, at least in a first approximation. is

The standard deviation of the hopping rate in eq. 2.16 on the electric field axis

$$
\sigma_{N M}=\frac{\sqrt{2 \lambda_{N M} k_{B} T}}{e \Delta z_{N M}}
$$


The maximum rate for the movement of the charge carrier $M \rightarrow N$ is obtained when $q E \Delta z_{N M}+\Delta E_{N M}+\lambda_{N M}=0$, while significant rates (in proportion) are obtained when the electric field $E$ is oscillating of $\pm 3 \sigma_{N M}$. At the extremes of this interval the electron hopping rate in the $\mathrm{z}$ direction is reduced roughly by $99.3 \%$ of its initial value, thereby drastically reducing conduction. If $\Delta E_{N M}$ belongs to the interval $\left(-\lambda_{N M}-3 \sigma_{N M},-\lambda_{N M}+3 \sigma_{N M}\right)$ we will express it as

$$
q E \Delta z_{N M} \bumpeq-\Delta E_{N M}-\lambda_{N M} .
$$

or even

$$
q E \Delta z_{N M}+\Delta E_{N M}+\lambda_{N M} \bumpeq 0 .
$$

The symbol $\bumpeq$ assumes thus a precise meaning in this context, it specifies that the first member of the equation belongs to the interval identified by the second member $\pm 3 \sigma_{N M}$. Formulas are thus more compact, but reader should keep in mind that the standard deviation $\sigma_{N M}$ is implicit in the notation used. The fields inside the interval specified by eq. 2.19 favor conduction in Marcus Theory. The range of the electric fields under the gaussian is the region in which the charge hopping has a significant probability to occur (with respect to the peak). The first half of this region, before the gaussian peak, where the probability is increasing with the field, is usually referred in the literature as correlation region [64]. After the gaussian peak, when the probability decrease with the field is referred to as the inverse Marcus region. Both these regions combined are called the active region and can be identified as the region in between two or three $\sigma_{N M}$ from the peak of the gaussian (Figure 2.2). The region before correlation, when the field is too low

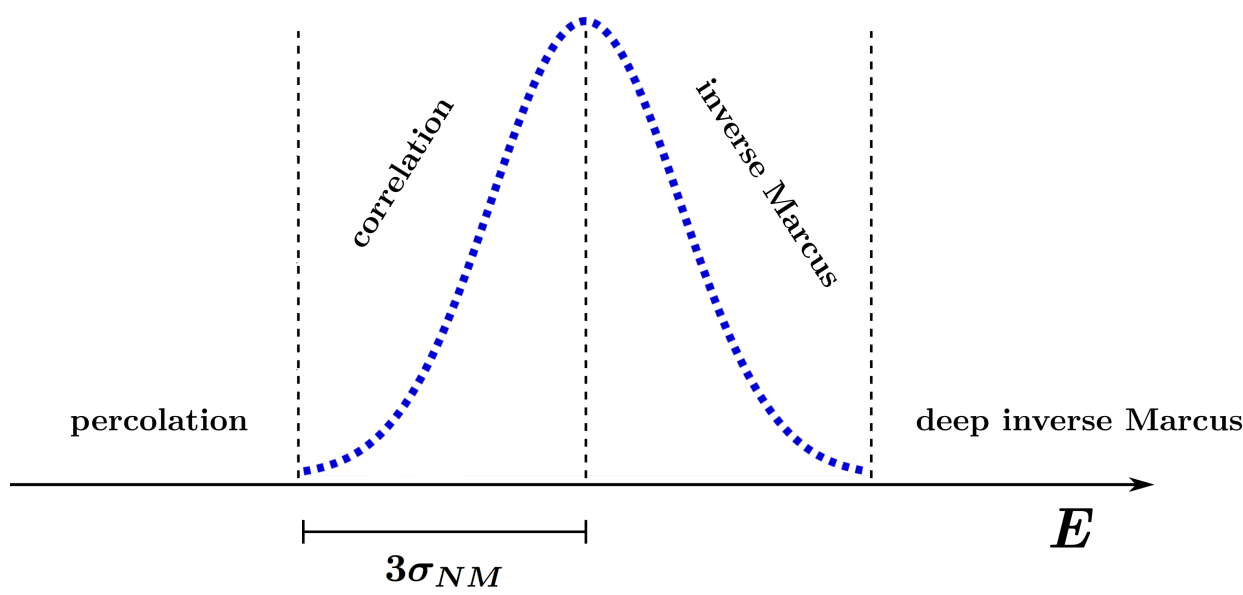

Figure 2.2. The different regions for an efficiency gaussian: percolation, correlation, inverse Marcus region and deep inverse Marcus region.

to influence the evolution of the system, is called percolation region. This name refers to the pioneering work of Bässler [35] in which he found that at small fields the percolation paths in the material are dominant for the charge movement, i.e. 
the field is too low to produce a real drift. The region in which the field is too strong and thus inhibits conduction in Marcus theory is called the deep inverse Marcus region. The illustration in Figure 2.3 is referred as efficiency diagram and represents the dependence of hopping rates of a single charge on the external electric field. The $3 \sigma_{N M}$ interval is projected on the field axis as a guide for the

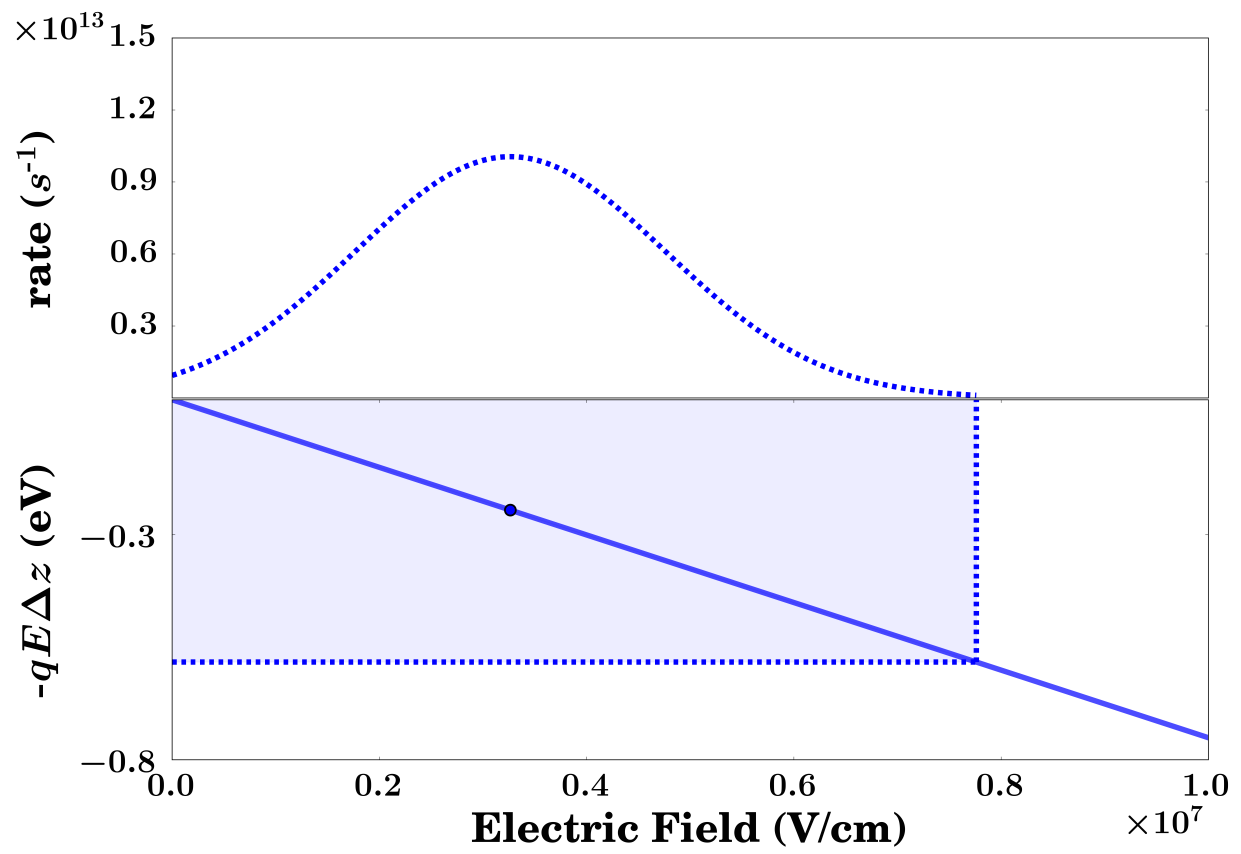

Figure 2.3. Efficiency diagram for an electron in the bulk.

eyes. This can be used to estimate the location of the different regions of mobility at equilibrium in a single material, as explained in Paper III. For an electron in the bulk we can write

$$
-e E \Delta z \bumpeq-\left(\Delta E_{\mathrm{bulk}}^{\mathrm{e}}+\lambda_{\mathrm{bulk}}^{\mathrm{e}}\right)
$$

where $\Delta z$ is the average layer spacing in the direction of the field, $\Delta E_{\text {bulk }}^{\mathrm{e}}$ and $\lambda_{\text {bulk }}^{\mathrm{e}}$ are the average difference in energy and reorganization energy in the bulk, respectively. An estimation of $\Delta E_{\text {bulk }}^{\mathrm{e}}$ has to be made, considering that the charge carrier has been relaxed in the lower tail of the DOS (in other words, the system reached the equilibrium of the Markov chain [35]). In general this difference is dependent from the correlation of the energetic landscape as shown in Paper I, but for an order of magnitude estimation a model with identically independently distributed gaussians can serve the purpose and the energy difference can be estimated as the standard deviation of the DOS, $\Delta E_{b u l k}^{\mathrm{e}} \sim \sigma_{D O S}$. An example of efficiency diagrams calculated for different contributions can be found in Paper III, 
illustrating how the DOS and the polarization of the environment determine the position and the width of the active region on the electric field axis.

\subsection{CT state splitting diagram}

We introduced in the previous section the efficiency diagram. This diagram illustrates the dependency of Marcus hopping rates on the field in the case of a single charge carrier. In the case of multiple charge carriers the diagram is expected to change, due to carrier-carrier interactions affecting the $\Delta E_{N M}$ of eq. 2.16. A case of particular interest is the CT state at the interface. In this situation an electron on an acceptor molecule and a hole on a donor molecule are bonded at the interface. Let us place the interface in the $x y$ plane with an external electric field acting in the $-z$ direction as in Figure 2.4. Two events are of particular interest. One is the CT state split, when the electron and the hole split apart far from each other and another is the charge carrier collection, this means that the particular charge carrier under consideration split and reached the respective end of the box. Four efficiency diagrams can be drawn to analyze this situation. At first we can

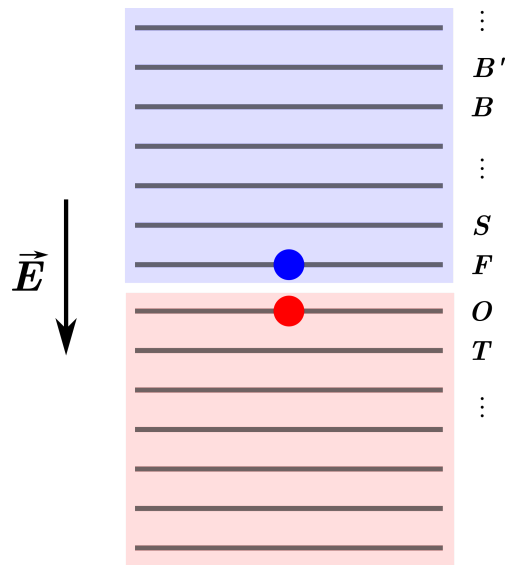

Figure 2.4. Illustration of the layers of the system. At the interface, for the acceptor (in blue), we note the first (F) and second (S) layer. For the donor (in red), we note layer minus one $(\mathrm{O})$ and minus two $(\mathrm{T})$. Two consecutive bulk layers are noted B and B'. Reproduced from Ref. [65] with permission from the Royal Society of Chemistry.

focus on the movement of the electron, keeping the hole still. When the electron is at the interface, the difference in energy for the electron hopping from the first to the second layer of molecules (in the opposite direction of the field) is

$$
\Delta E_{F_{0} S_{0}}^{\mathrm{e}}=\Delta E_{F_{0} S_{0}}^{\mathrm{e}-\mathrm{int}}+\Delta E_{F_{0} S_{0}}^{\mathrm{e}-\mathrm{h}},
$$

i.e. can be divided in the contribution of the interface $\Delta E^{\mathrm{e}-\mathrm{int}}$ and the attraction of the hole $\Delta E^{\mathrm{e}-\mathrm{h}} . F$ and $S$ symbolize that the electron is hopping from first 
to second layer and the 0 at the subscript specifies that this hop is happening between the ground states. $\Delta E_{F_{0} S_{0}}^{\mathrm{e}-\mathrm{int}}$ is composed by the shift of the energy levels of the molecules due to their geometrical deformation at the interface $(\Delta \epsilon)$, and by the electrostatic interaction of the interface with the charge carrier due to permanent atomic charges $\left(\Delta E^{\text {perm }}\right)$ and induced dipoles $\left(\Delta E^{\text {ind }}\right)$, all terms that can be calculated as will be detailed in Section 3.1. With the considerations made

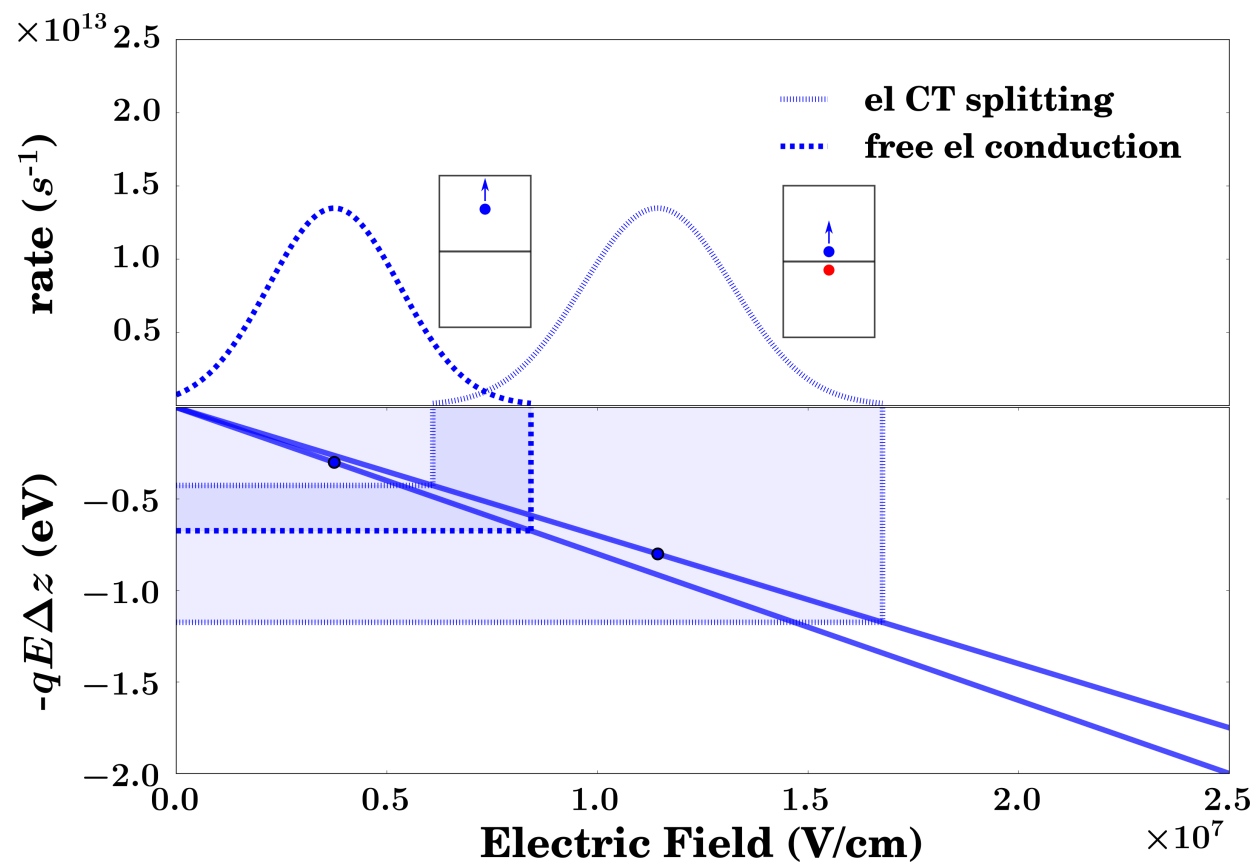

Figure 2.5. Efficiency diagram for the electron (for conduction close to and away from the interface). Reproduced from Ref. [65] with permission from the Royal Society of Chemistry.

in eq. 2.21 we can write the Marcus rate for an electron passing from first to second layer as a function of the external electric field

$w_{F_{0} S_{0}}^{\mathrm{e}}=\frac{2 \pi}{\hbar}\left|H_{F_{0} S_{0}}^{\mathrm{e}}\right|^{2} \sqrt{\frac{1}{4 \pi k_{\mathrm{B}} T \lambda_{F_{0} S_{0}}^{\mathrm{e}}}} \exp \left(-\frac{\left(-e E \Delta z_{F S}^{\mathrm{A}}+\Delta E_{F_{0} S_{0}}^{\mathrm{e}-\mathrm{int}}+\Delta E_{F_{0} S_{0}}^{\mathrm{e}-\mathrm{h}}+\lambda_{F_{0} S_{0}}^{\mathrm{e}}\right)^{2}}{4 \lambda_{F_{0} S_{0}}^{\mathrm{e}} k_{\mathrm{B}} T}\right)$

with $\Delta z_{F S}^{\mathrm{A}}$ being the distance between first and second layer of the acceptor in the $z$ direction (that does not depend on which molecular energy levels we are considering). The standard deviation of eq. 2.22 is readily calculated as

$$
\sigma_{F_{0} S_{0}}^{\mathrm{e}}=\frac{\sqrt{2 k_{\mathrm{B}} T \lambda_{F_{0} S_{0}}^{\mathrm{e}}}}{e \Delta z_{F S}^{\mathrm{A}}}
$$

Equation 2.22 represents the efficiency of the process considered (the electron splitting the CT state by passing from first to second layer) as a function of the 
external electric field. With the symbol defined in eq. 2.19, this can be written concisely as

$$
-e E \Delta z_{F S}^{\mathrm{A}} \bumpeq-\left(\Delta E_{F_{0} S_{0}}^{\mathrm{e}-\mathrm{int}}+\Delta E_{F_{0} S_{0}}^{\mathrm{e}}\right)-\lambda_{F_{0} S_{0}}^{\mathrm{e}} .
$$

Another interesting situation is when the electron is far from the interface (in the bulk). The hole is too far to interact with the electron, thus $\Delta E_{\text {bulk }}^{\mathrm{e}}$. This situation is slightly different from eq. 2.20 , since we are not interested in the equilibrium of the Markov chain (see Chapter 4), when the charge carrier has relaxed in the tail of the DOS [35]. In this case we will neglect the energy difference between two neighbouring sites (since it averages to 0 when the system is in an intermediate relaxation in the DOS). This strategy has been demonstrated to work very well when interested in the CT splitting at the interface, as in Papers V and VI. The average distance of two layers in the bulk in the $\mathrm{z}$ direction $\Delta z_{\text {bulk }}^{\mathrm{A}}$ is in general different from $\Delta z_{F S}^{\mathrm{A}}$ due to the deformations at the interface. The interface will indeed usually introduce an extra stress on the molecule leading to an increased compression of the interfacial layers (for example see [66]). The Marcus rate for a free electron moving in the bulk thus results

$$
w_{\text {bulk }}^{\mathrm{e}}=\frac{2 \pi}{\hbar}\left|H_{\text {bulk }}^{\mathrm{e}}\right|^{2} \sqrt{\frac{1}{4 \pi k_{\mathrm{B}} T \lambda_{\mathrm{bulk}}^{\mathrm{e}}}} \exp \left(-\frac{\left(-e E \Delta z_{\mathrm{bulk}}^{\mathrm{A}}+\lambda_{\mathrm{bulk}}^{\mathrm{e}}\right)^{2}}{4 \lambda_{\mathrm{bulk}}^{\mathrm{e}} k_{\mathrm{B}} T}\right)
$$

whose standard deviation is

$$
\sigma_{\mathrm{bulk}}^{\mathrm{e}}=\frac{\sqrt{2 k_{\mathrm{B}} T \lambda_{\mathrm{bulk}}^{\mathrm{e}}}}{e \Delta z_{\mathrm{bulk}}^{\mathrm{A}}}
$$

For the electron movement then we can finally obtain the pair of equations

$$
\begin{aligned}
-e E \Delta z_{F S}^{\mathrm{A}} \bumpeq-\left(\Delta E_{F_{0} S_{0}}^{\mathrm{e}-\mathrm{nt}}+\Delta E_{F_{0} S_{0}}^{\mathrm{e}}\right)-\lambda_{F_{0} S_{0}}^{\mathrm{e}} \\
-e E \Delta z_{\text {bulk }}^{\mathrm{A}} \bumpeq-\lambda_{\text {bulk }}^{\mathrm{e}} ;
\end{aligned}
$$

representing the system in the two situations considered, when the electron is at the interface or in the bulk. Equation 2.27 is related to the efficiency of the CT state splitting initiated by the electron, while equation 2.28 is related to the transport of the free electron to the electrode. When both equations are satisfied simultaneously we have an efficient CT state dissociation (initiated by the electron) and free electron transport in the acceptor phase. Figure 2.5 illustrates these facts with the efficiency diagrams for the electron. The $3 \sigma$ tolerances are illustrated both on the energy and on the field axis.

Analogous considerations can be made for the hole and two new constraints can be found, regarding the efficient CT state splitting (initiated by the hole) and the free hole transport to the electrode

$$
\begin{aligned}
-e E \Delta z_{O T}^{\mathrm{D}} \bumpeq-\left(\Delta E_{O_{0} T_{0}}^{\mathrm{h}-i n t}+\Delta E_{O_{0} T_{0}}^{\mathrm{h}-\mathrm{e}}\right)-\lambda_{O_{0} T_{0}}^{\mathrm{h}} \\
-e E \Delta z_{\text {bulk }}^{\mathrm{D}} \bumpeq-\lambda_{\text {bulk }}^{\mathrm{h}} ;
\end{aligned}
$$

$O$ and $T$ represents the first and second layer for the hole (Figure 2.4). When referring to the hole, $\mathrm{h}$ is present in the superscript, thus the subscript 0 refers 
to the ground state of the hole, i.e. the HOMO in $O_{0}$ and $T_{0}$. The conditions imposed by equations 2.29-2.30 can be illustrated with the efficiency diagrams for the hole, Figure 2.6. Joining now the efficiency diagrams for the holes and electrons

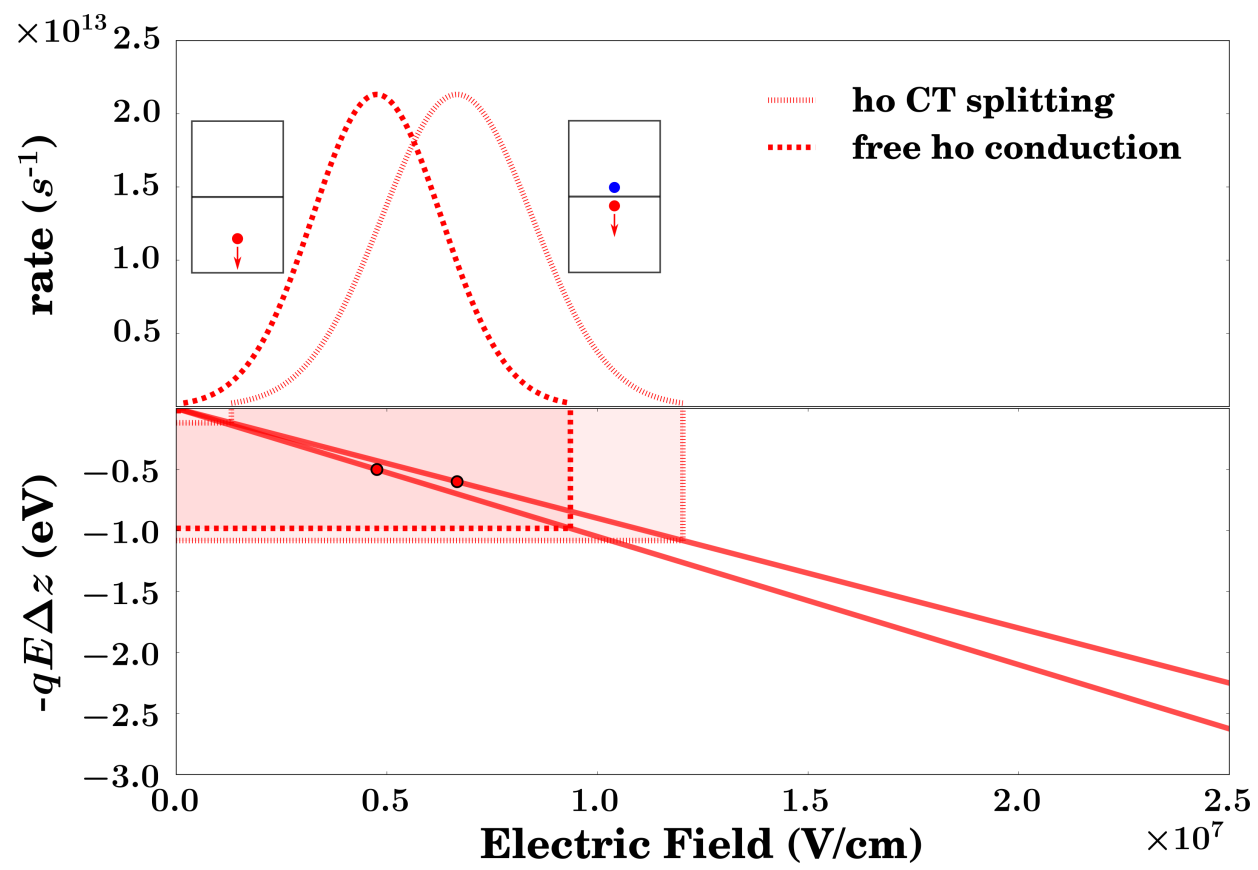

Figure 2.6. Efficiency diagram for the hole (for conduction close to and away from the interface). Reproduced from Ref. [65] with permission from the Royal Society of Chemistry.

we obtain what we will call the CT state splitting diagram, Figure 2.7. In this diagram we find summarized the dependency of the rates in function of the electric field. To obtain a good charge collection rate, the gaussians of the CT state split and the free charge carrier conduction should have a significant overlap for at least one of the two charges (electron or hole). In this way it will be possible to find a field at which the charge carriers can initiate the CT state splitting and also can subsequently be collected at the electrode (Figure 2.8). This condition can be expressed requiring that the peaks of the two gaussians will be nearer than the smallest of the widths (see also [65]), i.e. for an electron

$$
\frac{\left|\Delta E_{F_{0} S_{0}}^{\mathrm{e}}\right|}{e \Delta z_{F S}^{\mathrm{A}}} \lesssim 3\left(\sigma_{F_{0} S_{0}}^{\mathrm{e}}+\sigma_{\text {bulk }}^{\mathrm{e}}\right)
$$

while for the hole we obtain

$$
\frac{\left|\Delta E_{O_{0} T_{0}}^{\mathrm{h}}\right|}{e \Delta z_{O T}^{\mathrm{D}}} \lesssim 3\left(\sigma_{O_{0} T_{0}}^{\mathrm{h}}+\sigma_{\text {bulk }}^{\mathrm{h}}\right) .
$$




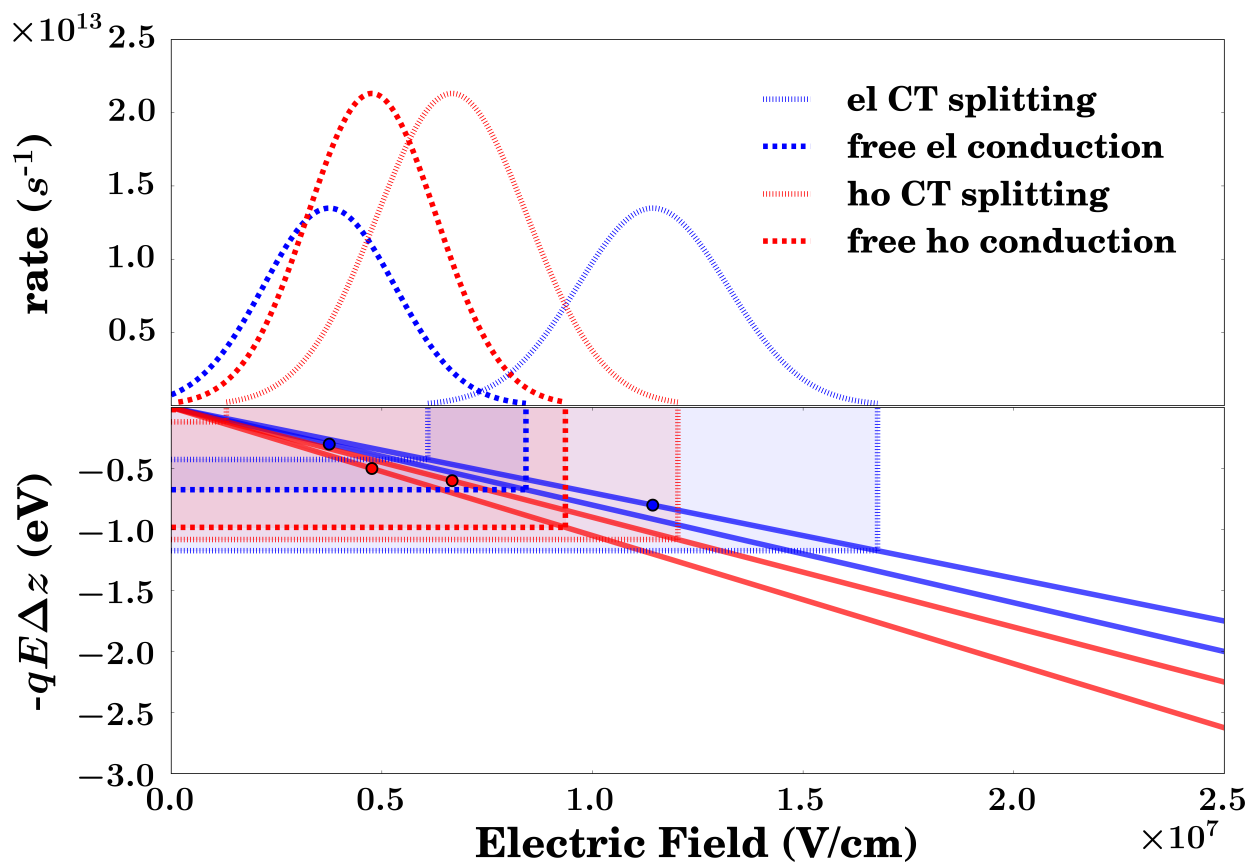

Figure 2.7. CT state splitting diagram. Reproduced from Ref. [65] with permission from the Royal Society of Chemistry.

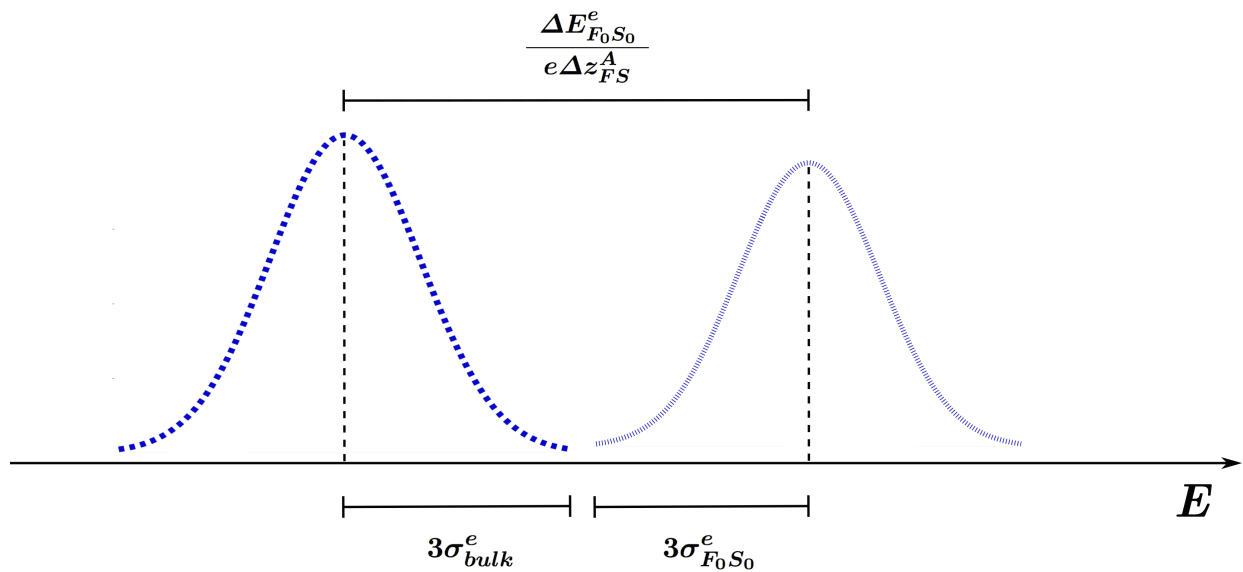

Figure 2.8. Illustration of the two gaussians for electron in the CT state and free electron conduction. The factors promoting or impeding the overlap are noted on the figure. Reproduced from Ref. [65] with permission from the Royal Society of Chemistry.

In the beginning the system is represented by the two CT splitting gaussians, once the CT state has been split, the system will happen to be in an intermediate state between CT splitting and free conduction gaussian for both of the charge carriers. 
Ultimately we would like both charges to be able to move at the same fields, i.e. we require an overlap between the two free conduction gaussians

$$
\left|\frac{\lambda_{\text {bulk }}^{\mathrm{e}}}{e \Delta z_{\text {bulk }}^{\mathrm{A}}}-\frac{\lambda_{\text {bulk }}^{\mathrm{h}}}{e \Delta z_{\text {bulk }}^{\mathrm{D}}}\right| \lesssim 3\left(\sigma_{\text {bulk }}^{\mathrm{e}}+\sigma_{\text {bulk }}^{\mathrm{h}}\right) .
$$

If these conditions can be met (eq. 2.33 and one of 2.31 or 2.32), it means that the two materials under consideration are well suitable to be used together in an organic solar cell.

The analysis presented so far, involves only ground states and thus it is focused on the cold CT state splitting. The role of the hot CT state (i.e. electron and/or hole on a higher energetic level) is highly debated in the literature. In particular the role ot the excess energy (resulting from absorption of a photon with energy higher than the HOMO-LUMO gap) is still unclear. Several studies suggest how the excess energy is favouring the CT state dissociation, opening up new energetic paths (on higher energy levels). [18,67-72] Other studies, focused on polymerfullerene solar cells, instead point out how the exciton dissociation in these systems is independent on the excess energy and thus they seem to suggest how any excess energy will be quickly lost, till a cold CT state has been reached. [19, 73, 74] It is not difficult to imagine how higher energy levels will modify the diagram presented in Figure 2.7. First of all, the transfer integrals $H_{F_{m} S_{n}}$ involving higher energy levels are expected to increase in magnitude with increasing $m$ and $n$ due to the increasing delocalization of the molecular orbitals. This is true in average, even if some oscillatory behaviour due to the nodes of the wavefunctions might be present when increasing $m$ and $n$. We will then expect some gaussians with higher amplitude that will corresponds to high probability paths, if at the right field. Involving higher energy levels will also change the electronic distribution on the molecule and thus, even if the total charge will still be +1 for hole and -1 for an electron, the higher order terms of the charge distribution will change (dipole, quadrupole, etc.) In a first approximation we neglect these contributions and thus consider for the electron

$$
\Delta E_{F_{m} S_{n}}^{\mathrm{e}} \approx \Delta E_{F_{0} S_{0}}^{\mathrm{e}}-\Delta \epsilon_{F_{0} S_{0}}^{\mathrm{e}}+\Delta \epsilon_{F_{m} S_{n}}^{\mathrm{e}}
$$

where the effect of considering excited levels can be seen mainly in the differences $\Delta \epsilon_{F_{m} S_{n}}^{\mathrm{e}}$ and the term $\Delta E_{F_{0} S_{0}}^{\mathrm{e}}-\Delta \epsilon_{F_{0} S_{0}}^{\mathrm{e}}$ represents exclusively the contributions of permanent charges and induced dipoles on an electron in the LUMO (see eq. 2.21). For a hole the same reasoning leads to

$$
\Delta E_{O_{m} T_{n}}^{\mathrm{h}} \approx \Delta E_{O_{0} T_{0}}^{\mathrm{h}}-\Delta \epsilon_{O_{0} T_{0}}^{\mathrm{h}}+\Delta \epsilon_{O_{m} T_{n}}^{\mathrm{h}} .
$$

In this way we can understand how the difference in energy $\Delta \epsilon$ contributes to the shifting of the peak of the hot CT state splitting gaussians and thus will be possible to find some excited levels with good overlap with the free conduction gaussian or with other CT splitting gaussians, e.g. $F_{m} \rightarrow S_{n}$ followed by $S_{m} \rightarrow R_{p}$, where $R$ represents here a consecutive layer and $p$ another possible energetic level. This definitely complicates the CT state splitting diagram, including all the possible competing rates, but it allows to make an approximate prediction on the chain of events leading to the CT split. 


\section{CHAPTER 3}

\section{Marcus Parameters}

As already stressed in Section 1.3, a key component of our work is to consider a realistic morphology for organic solar cells, without assuming any crystallinity in the material. As a consequence, for every nearby molecule in a certain cut-off, we need to calculate one hopping rate for each possible molecular orbital on that molecule (eq. 2.16)

$$
w_{M N}=\frac{2 \pi}{\hbar}\left|H_{N M}\right|^{2} \sqrt{\frac{1}{4 \pi k_{B} T \lambda_{N M}}} \exp \left(-\frac{\left(-q \vec{E}_{\mathrm{ext}} \cdot \Delta \vec{r}_{N M}+\Delta E_{N M}+\lambda_{N M}\right)^{2}}{4 \lambda_{N M} k_{B} T}\right) .
$$

Each hop from $M=(i, m)$ to $N=(j, n)$ necessitates of: the energy difference $\Delta E_{N M}$, the transfer integral $H_{N M}$ and the reorganization energy $\lambda_{N M}$. The temperature $T$ and the external electric field $\vec{E}_{\text {ext }}$ are instead global parameters given as input of the simulation. How to model or calculate the parameters needed for the hopping rates will be argument of discussion of the present chapter.

\subsection{Site Energies and Field Contribution}

The energy $\Delta E_{N M}=u_{N}-u_{M}$ is the difference between the energies of the two orbitals considered ( $M$ and $N$ ). The single site energy $u_{M}$ can be calculated in several ways. One of the simplest one is assuming $u_{M}$ to be distributed as a gaussian and sample the energies independently from this same gaussian distribution for each molecular orbital level $M$ [35]. This is an approach that allows to recover a realistic density of states (DOS) and the right qualitative mobility temperature dependence, but it fails in modelling the local correlation of the energetic landscape that is needed to predict Poole-Frenkel mobility field dependence [64,75,76]. This latter is indeed deriving from dipole correlations $[75,76]$ as shown also in Paper I. 
The correlation of the energetic landscape thus arises naturally when considering electrostatic interactions between the extra charge carrier and the nearby molecules. In the rest of the Dissertation and in Papers II, III, V and VI, the site energy is then expressed as

$$
u_{M}=\epsilon_{M}+E_{M}^{p e r m-e l}(\underline{s})+E_{M}^{i n d-e l}(\underline{s}) .
$$

The first term $\epsilon_{M}$ in equation (3.2) is the orbital energy obtained from a QM calculation. Since these calculations are done for every molecule in the box, this value takes into account the energy shift caused by the conformational changes of the molecule in the bulk. The other terms in eq. 3.2 arise from the interaction of the charged molecule with its environment. They are dependent on the quantum state of the charged molecule, due to the extra charge on the orbital $M$. Two cut-offs are used for this task, leading to two different interaction sets of molecules: the interaction set for permanent charges $I(M)$ and the interaction set for polarization $I_{p}(M)$ (see Figure 3.1). The array $\underline{s}=\left(s_{1}, s_{2}, \ldots, s_{i}, s_{j}, \ldots\right)$ is composed by the

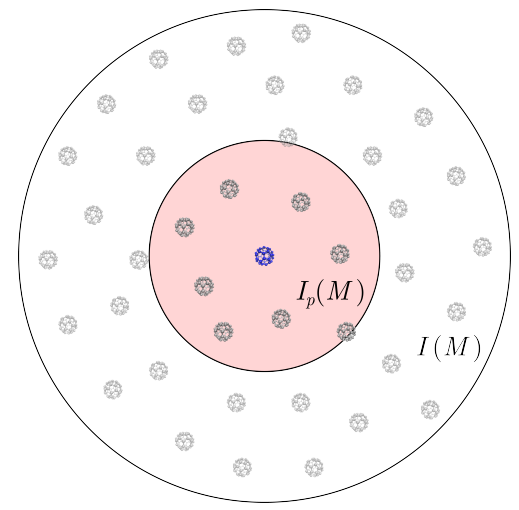

Figure 3.1. The interaction sets used for Coulombic interactions $I(M)$ and polarization $I_{p}(M)$, for a charge carrier situated on the molecular orbital $M$.

quantum states of every molecule in the system. As a consequence of a hopping process a molecule will change quantum state, so that the state of the system $\underline{s}$ varies over time. For the cases considered in Paper I-III, a molecule can only be neutral $(n)$ or charged $(c)$, where charged means different things depending on the molecule considered. For D molecules charged means one extra hole in the HOMO level, while for A molecules means on extra electron in the LUMO level. The atomic charges and polarizabilities of a molecule will change according to the molecular quantum state during the simulation. In the $\mathrm{C}_{60}$ example, when an electron moves from the molecular level $M=(i, L U M O)$ to the molecular level $N=(j, L U M O)$, the system passes from $\underline{s_{1}}=(n, n, \ldots, c, n, \ldots)$ to $\underline{s_{2}}=(n, n, \ldots, n, c, \ldots)$ (Figure 3.2 on the top). The second term in equation (3.2) represents the permanent electrostatic energy

$$
E_{M}^{p e r m-e l}(\underline{s})=\frac{1}{4 \pi \epsilon_{0}} \sum_{a \in i} \sum_{\substack{b \in h \\ h \in I(M)}} \frac{q_{a}\left(s_{i}\right) q_{b}\left(s_{h}\right)}{\left|\vec{r}_{a}-\vec{r}_{b}\right|}
$$



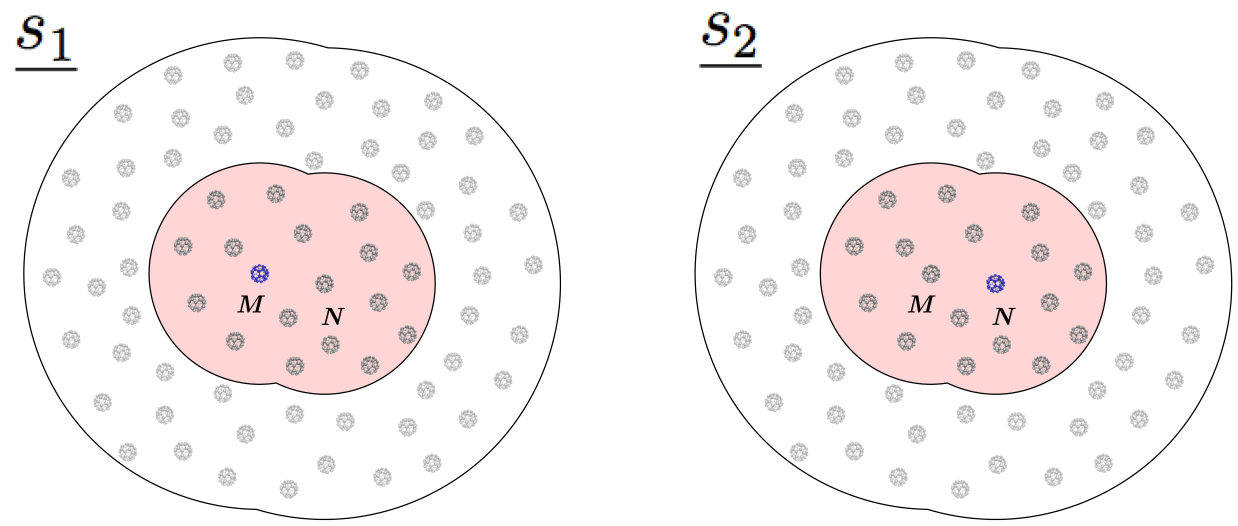

Figure 3.2. Illustration of two quantum states $s_{1}$ and $s_{2}$ whose difference is a charge hopping. Reprinted with permission from JCTC 2016, 12, 812. Copyright 2016 American Chemical Society.

where $h$ is a molecule belonging to the interaction set $I(M)$ and $a, b$ are atoms, whose positions are denoted $\vec{r}_{a}$ and $\vec{r}_{b}$ respectively. This sum is based on the atomic charges and thus includes the contribution of molecular permanent dipoles, quadrupoles, etc. The atomic charge $q_{a}$ on the atom $a$ depends on the quantum state of molecule $i$, which the atom belongs to. This is why it has been denoted as $q_{a}\left(s_{i}\right)$. The third term of (3.2) is the energy due to polarization. Polarization in an organic material arises from two different contributions, the reorganization of the permanent multipoles of nearby molecules (through the movement of their nuclei) and the polarization of the nearby electronic clouds. The first contribution is slow compared to the timescale of a charge carrier hopping and will be neglected in our model. The second contribution is instead faster and can be accounted for through atomic polarizabilities and induced dipoles. The resulting polarization energy is

$$
E_{M}^{i n d-e l}(\underline{s})=\frac{1}{4 \pi \epsilon_{0}} \sum_{a \in i} \sum_{\substack{b \in h \\ h \in I_{p}(M)}} \frac{q_{a}\left(s_{i}\right) \overrightarrow{p_{b}}(\underline{s}) \cdot\left(\overrightarrow{r_{a}}-\overrightarrow{r_{b}}\right)}{\left|\overrightarrow{r_{a}}-\overrightarrow{r_{b}}\right|^{3}}
$$

where $p_{b}(\underline{s})$ represents the induced dipole at the atom $b$, given that the nearby molecules are in the state $\underline{s}$. Since we want to avoid border effects, the interaction set $I_{p}(M)$ chosen for the polarization is always smaller than the one chosen for the permanent charges $I(M)$. The dipoles inside $I_{p}(M)$ are calculated depending on the permanent charges of all the molecules in the interaction set $I(M)$.

In the rest of the Dissertation and in the papers we use a particular nomenclature specifying the energy assignment method that has been used. ' $O$ ' stands for the molecular orbital energies, ' $\mathrm{C}$ ' for the Coulombic interactions from permanent charges (3.3) and ' $\mathrm{P}$ ' for the energy corrections resulting from polarization (3.4). $\mathrm{O}$ is used in case the energy is composed only by the QM orbital energies, OC when the energy is composed by the orbitals corrected by interactions with nearby permanent charges and OCP when all the three contributions are included. These 
three terms contribute to what will be referred as density of states (DOS) for an extra charge carrier in the material. An illustration of the DOS for an electron in disordered $\mathrm{C}_{60}$ considering different contributions is reported in Figure 3.3

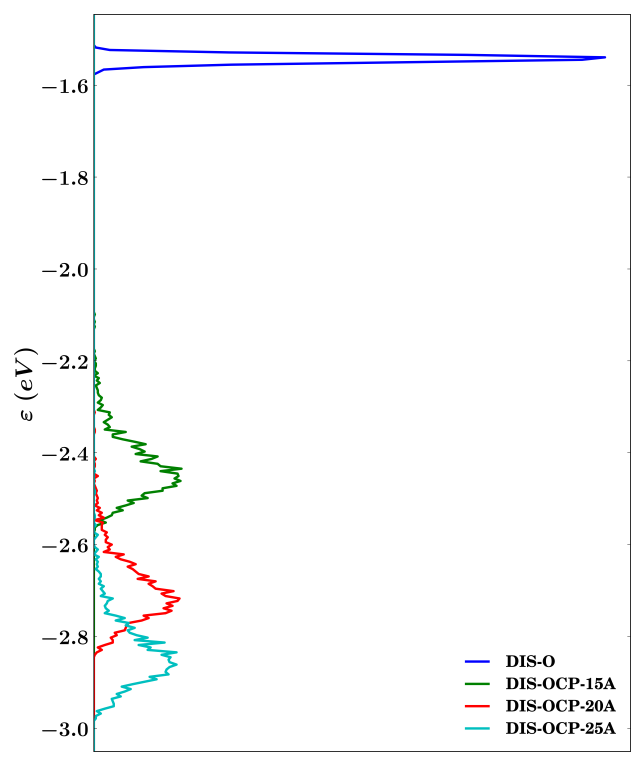

Figure 3.3. $\mathrm{O}, \mathrm{OC}$ and $\mathrm{OCP}$ density of state for a disordered $\mathrm{C}_{60}$ system. More information on how $\mathrm{C}$ and $\mathrm{P}$ have been calculated can be found in Paper II. Reprinted with permission from JCTC 2016, 12, 812. Copyright 2016 American Chemical Society.

Finally, the interaction of the charge carrier with the external electric field will be considered. $\Delta \vec{r}_{N M}$ in eq. 3.1 can be written as

$$
\Delta \vec{r}_{N M}=\Delta \vec{r}_{j i}=\frac{\sum_{b \in j} q_{b}(s) \overrightarrow{r_{b}}-\sum_{a \in i} q_{a}(s) \overrightarrow{r_{a}}}{e}
$$

leading to the field contribution

$$
-e \vec{E}_{\mathrm{ext}} \cdot \Delta \vec{r}_{N M}=-\vec{E}_{\mathrm{ext}} \cdot\left(\sum_{b \in j} q_{b}(s) \overrightarrow{r_{b}}-\sum_{a \in i} q_{a}(s) \overrightarrow{r_{a}}\right)
$$

representing the interaction of atomic charges with the external electric field $\vec{E}_{\text {ext }}$.

\subsection{Transfer Integrals}

One of the simplest methods to calculate the transfer integrals is called the energysplitting-dimer (ESD) method $[77,78]$. When the molecules are well $\pi$-stacked, an extra charge will be considered equally distributed over couples of nearby molecules and the energy difference between $M$ and $N$ is approximately equal to $2 H_{N M}$. 
Therefore, if the geometries of the molecules are known, the transfer integral can be calculated. To simplify the calculations, the geometries are often approximated as geometries of a neutral dimer. This method works for organic semiconductors exhibiting a well $\pi$-stacked regular structure, but fails where disordered is present in the system.

Another basic method is the exponential decay or distance dependent (DD) transfer integral

$$
H_{N M}=H_{0} \exp \left(-\alpha r_{N M}\right),
$$

here the transfer integral is considered to be exponentially decaying with the distance $r_{N M}$ between the two molecular orbitals $M$ and $N . H_{0}$ and $\alpha$ are two parameters that have to be chosen ad hoc for each molecule. This method works well in first approximation but fails in capturing anisotropies in the transfer integrals distribution.

A first method capturing the directionality of the transfer integral distribution is the distance orientation dependent (DOD) transfer integral, using a weighted Mulliken formula [79] for carbon atoms [80], based on the approximation that only p-electrons contributes to the transfer integral. This approach has been used in ref. [44] and Paper I. It provides a quick and approximated formula but still gives a dependence of the transfer integrals on the relative orientation of two neighbouring molecules.

If wishing for a better description, the transfer integrals can be directly calculated from the definition, as off diagonal elements of the Hamiltonian. Three QM calculations are needed for this purpose: one for the pair of molecules considered $i j$ (or dimer $d$ ) and two for the single molecules $i$ and $j$. The Fock matrix of the pair is then projected on the single molecular orbitals basis (the basis of the molecular orbitals of the two single molecules) and the transfer integrals $\left|H_{N M}\right|$ can be extracted as the non-diagonal elements of this rotated Fock matrix in the single molecular orbitals basis. This is known in literature as the projection method and it is the method used in Papers II-VI. It was originally formulated for ZINDO [81] and it has been subsequently extended [82] for more sophisticated QM calculations that do not assume orthogonality of atomic orbitals. For each calculation $(i, j$ and d) we obtain a set of wavefunctions

$$
\left|\psi_{m}\right\rangle=\sum_{\nu} \varphi_{\nu} C_{\nu m}
$$

expanded in the atomic basis set $\left\{\varphi_{\nu}\right\}$ and identified by the quantum numbers of the particular orbital level $m$. The transfer integral from $M=(i, m)$ to $N=(j, n)$ can be written as

$$
H_{N M}=\left\langle\psi_{m}^{i}|H| \psi_{n}^{j}\right\rangle=\sum_{r, s}\left\langle\psi_{m}^{i} \mid \psi_{r}^{d}\right\rangle\left\langle\psi_{r}^{d}|H| \psi_{s}^{d}\right\rangle\left\langle\psi_{s}^{d} \mid \psi_{n}^{j}\right\rangle
$$

where the completeness of the eigenfunctions of the dimer $\left\{\left|\psi_{r}^{d}\right\rangle\right\}$ have been used. The transfer integrals are calculated assuming the dimer system $d$ in the vacuum, meaning that the Hamiltonian from which extract the transfer integral is the dimer Hamiltonian, i.e.

$$
\left\langle\psi_{r}^{d}|H| \psi_{s}^{d}\right\rangle=\epsilon_{r}^{d} \delta_{r s},
$$


where $\epsilon_{r}^{d}$ is the eigenvalue associated to the eigenfunction $\left|\psi_{r}^{d}\right\rangle$ and $\delta_{r s}$ is the Kronecker delta. Substituting eq. 3.8 and 3.10 in eq. 3.9 we arrive at

$$
H_{N M}=\Pi^{i d} F^{d}\left(\Pi^{j d}\right)^{T} .
$$

Here $F^{d}$ is the Fock matrix of the dimer composed by the energies $\left\{\epsilon_{r}^{d}\right\}$, while the meaning of the matrices $\Pi^{i d}$ and $\Pi^{j d}$ will be discussed in the following. Let us assume there are $n_{i}$ atomic basis functions $\left\{\varphi_{\nu}^{i}\right\}$ for molecule $i$ and $n_{j}$ atomic basis functions $\left\{\varphi_{\mu}^{j}\right\}$ for molecule $j$, then there will be $n_{d}=n_{i}+n_{j}$ atomic basis functions $\left\{\varphi_{\chi}^{d}\right\}$ for the dimer and these basis functions can be rearranged such that the first $n_{i}$ functions of $\left\{\varphi_{\chi}^{d}\right\}$ are equal to $\left\{\varphi_{\nu}^{i}\right\}$, while the remaining $n_{j}$ atomic basis functions for the dimer are equal to $\left\{\varphi_{\mu}^{j}\right\}$. The matrices $\Pi^{i d}$ and $\Pi^{j d}$ have dimensions $n_{i} \times n_{d}$ and $n_{j} \times n_{d}$ respectively, and they take care of the projection of the eigenfunction of the dimer on the eigenfunctions of the single molecules and vice versa. They can be defined element-wise as

$$
\begin{aligned}
\Pi_{m r}^{i d} & =\left\langle\psi_{m}^{i} \mid \psi_{r}^{d}\right\rangle \\
\Pi_{n r}^{j d} & =\left\langle\psi_{n}^{j} \mid \psi_{r}^{d}\right\rangle .
\end{aligned}
$$

As expected from Supporting Information of Paper II, ZINDO underestimates the transfer integrals, expecially at greater distance where the diffuseness of the basis become more and more important. Nevertheless, it can capture the overall behaviour and it is a quite good approximation able to capture a qualitative behaviour of the transfer integrals on the relative orientation of the molecules. An illustration of the transfer integrals population calculated at the ZINDO, HF /6-31G and B3LYP/6-31G level in $\mathrm{PC}_{61} \mathrm{BM}$ (system of Paper III) is reported in Figure 3.4.

In all the methods introduced in this section, transfer integrals are evaluated excluding the surrounding molecules. However, several studies showed that the polarization can have a non negligible effect on the transfer integral $[28,29,31,83-85]$. For instance, Castet et al. showed by using a valence bond approach that the inclusion of polarization can decrease the value of transfer integrals by $10-15 \%$ for a hole in anthracene [85].

\subsection{Reorganization Energies}

The reorganization energy $\lambda$ is the energy released in all the structural adjustments of the system, after the charge hopping has occurred. It can be decomposed in two parts, an internal and an external reorganization energy

$$
\lambda=\lambda_{\text {int }}+\lambda_{\text {out }}
$$

The internal reorganization energy $\left(\lambda_{i n t}\right)$ is given by the internal geometry rearrangement of the molecule, after a charge is added or subtracted to the molecule. Let us consider a charge hopping from molecule $i$ to molecule $j$. The internal reorganization energy is the sum of the reorganization energy of the molecule $i$ 
Transfer Integrals for 'pcbm' molecules: from orbital 0 to orbital 0 (within a maximum edge distance of $6.00 \AA$ )

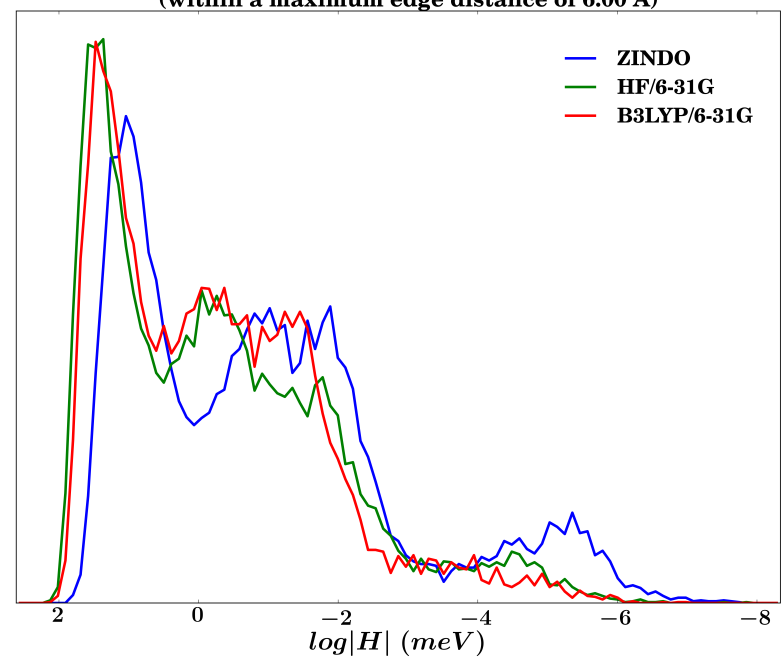

Figure 3.4. Transfer integral population in $\mathrm{PC}_{61} \mathrm{BM}$, calculated at different levels of theory.

that is passing from charged to neutral state and the reorganization energy of the molecule $j$ that is passing from neutral to charged state

$$
\lambda_{i n t}=\lambda_{i}^{c n}+\lambda_{j}^{n c} .
$$

These energies can be calculated performing quantum mechanical geometry reoptimizations on neutral and charged molecules and analyzing the energies obtained $[86,87]$ :

$$
\begin{gathered}
\lambda_{i}^{c n}=E_{i}^{n C}-E_{i}^{n N} \\
\lambda_{j}^{n c}=E_{j}^{c N}-E_{j}^{c C}
\end{gathered}
$$

where for example $E_{i}^{n C}$ is the energy of molecule $i$ in the geometrical conformation of the charged molecule $(C)$, but in the neutral electronic configuration $(n)$. Since geometry optimizations at the quantum mechanical level are computationally expensive, these calculations are done once for all for a single isolated molecule and the resulting $\lambda_{\text {int }}$ is used for all the molecules in the system.

The external reorganization energy $\left(\lambda_{\text {out }}\right)$ is given by the re-polarization of the environment after the charge carrier's jump. As for the single site energies, we neglect the rearrangement of the permanent nearby multipoles, i.e. we do not perform molecular dynamics during the KMC simulation. This structural rearrangement is much slower than both the wave functions polarization and the internal reorganization of the molecule (fast reorganization of covalent bonds). The polarization contribution is taken into account by means of induced dipoles on the 

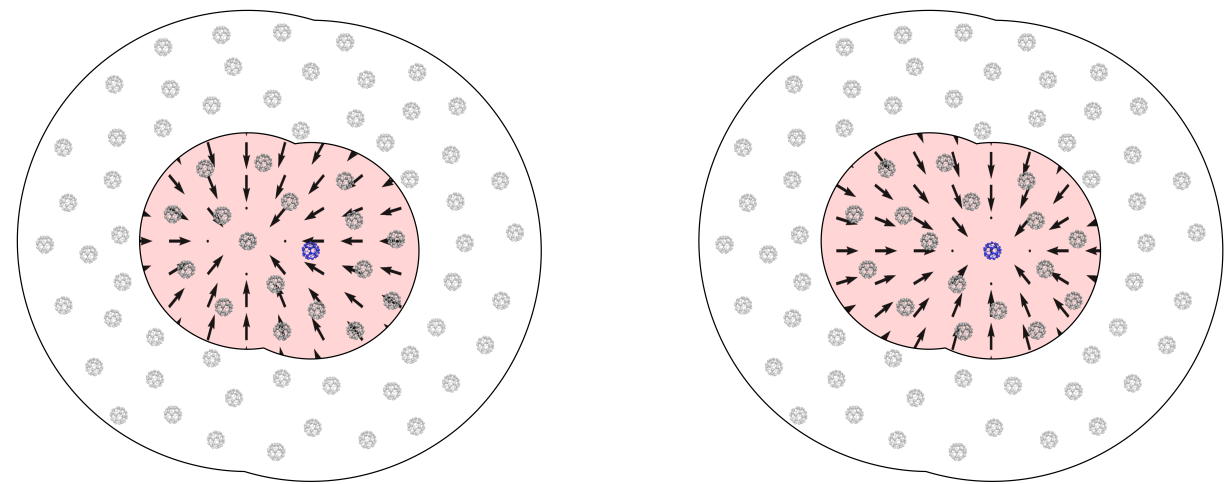

Figure 3.5. After the hop from $M$ to $N$, the situation before and after the reorganization. Reprinted with permission from JCTC 2016, 12, 812. Copyright 2016 American Chemical Society.

nearby molecules. Let us consider the energy contribution

$$
E_{M}^{i n d-e l}\left(\underline{s_{1}}, \underline{s_{2}}\right)=\frac{1}{4 \pi \epsilon_{0}} \sum_{a \in i} \sum_{\substack{b \in h \\ h \in I_{p}(M)}} \frac{q_{a}\left(s_{1 i}\right) \vec{p}_{b}\left(\underline{s_{2}}\right) \cdot\left(\overrightarrow{r_{a}}-\overrightarrow{r_{b}}\right)}{\left|\overrightarrow{r_{a}}-\overrightarrow{r_{b}}\right|^{3}}
$$

where as above $a$ and $b$ are atoms, orbital $M$ belongs to molecule $i$ and $h$ is a molecule in the polarization interaction set of $M$. This is the interaction energy between the charges on $i$ as given by the quantum state $s_{1}$ and the nearby dipoles induced by the quantum state $s_{2}$. When a jump from $M \overline{\text { to }} N$ occurs, the quantum states are modified from $\underline{s_{1}}$ to $\underline{s_{2}}$, i.e. the state of $i$ changes from charged to uncharged and the state of $j$ changes from uncharged to charged. An illustration of the states $\underline{s}_{1}$ and $\underline{s}_{2}$ with their respective induced dipoles are presented in Figure 3.5. The expression we are looking for has to describe the energy required for the external dipoles rearrangement, i.e. the difference between the energy of the new charge configuration in the old dipole arrangement (the jump happened but the structure did not relax yet) and the energy of the new charge configuration in the new dipole arrangement (system has re-polarized through dipoles reorganization). Consequently we can write the external reorganization energy as

$$
\lambda_{\text {out }}=E_{M}^{i n d-e l}\left(\underline{s_{2}}, \underline{s_{1}}\right)+E_{N}^{i n d-e l}\left(\underline{s_{2}}, \underline{s_{1}}\right)-E_{M}^{i n d-e l}\left(\underline{s_{2}}, \underline{s_{2}}\right)-E_{N}^{i n d-e l}\left(\underline{s_{2}}, \underline{s_{2}}\right) .
$$

The first and second terms are the energies given by the interaction of the atomic charges of molecules $i$ and $j$ in the state given by $\underline{s_{2}}$ (after the hop) with the induced dipoles given by the state $\underline{s}_{1}$ (before the hop). The remaining two terms are the energies of the interaction of the atomic charges of molecules $i$ and $j$ in the state given by $\underline{s}_{2}$ with the induced dipoles given by the same state $\underline{s}_{2}$ (final situation after the hop). Note that for the reorganization energy we neglect the energetic contribution between the electric field and the induced dipoles. We think this contribution is of secondary importance and we decided to neglect it (see more 


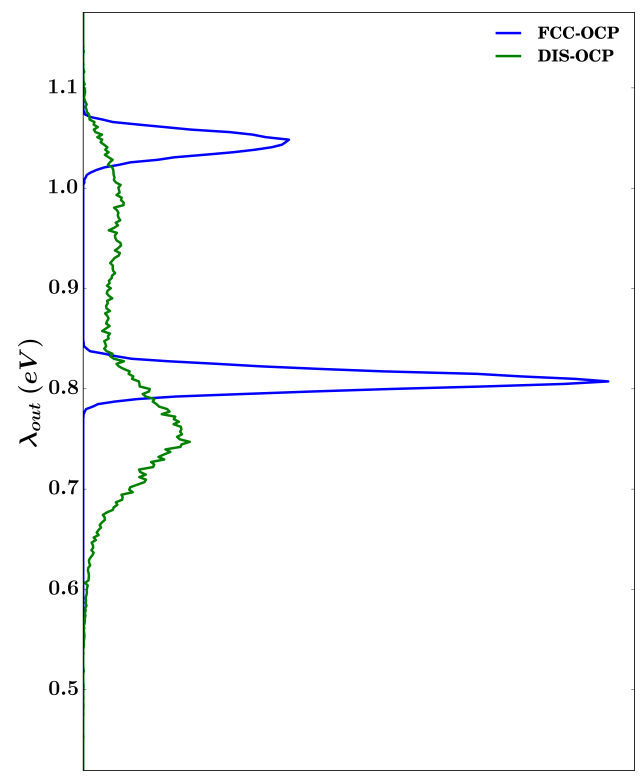

Figure 3.6. External reorganization in crystalline and disordered $\mathrm{C}_{60}$. Reprinted with permission from JCTC 2016, 12, 812. Copyright 2016 American Chemical Society.

in Paper II). Furthermore it can be demonstrated that the external reorganization energy defined by eq. 3.17 is independent from external electric field and additional charges, due to the linearity of the polarization model employed.

An illustration of the external reorganization energy in crystalline and disorder $\mathrm{C}_{60}$ (Paper II) is presented in Figure 3.6. In the crystalline system it is possible to identify two peaks, coming from first and second shell respectively, while in the disordered system these peaks are smeared out and merge together. The reader will notice how the external reorganization energy (calculated in this case with a cut-off of $20 \AA$ ) represents quite an important contribution. This comes from the fact that organic molecules are in general highly polarizable. If a full repolarization of the environment has to be taken into account after every hop is still matter of debate, see Chapter 5 and Papers II and III. 


\section{CHAPTER 4}

\section{Monte Carlo Simulations}

In the 1930s, Fermi used sampling to estimate quantities involved in controlled fission [88]. This sampling was performed by hand or using a mechanical device called "Fermiac". At the time of the Manhattan project in Los Alamos, John von Neumann and Stanislaw Ulam used computers to generate the system samples, getting results in a relatively fast and automatic way. Being secret, the work of von Neumann and Ulam had a code name, the name Monte Carlo, seems to refer to the Monte Carlo Casino in Monaco where Ulam's uncle would go to gamble borrowing money from relatives [89]. In 1949 Metropolis and Ulam published their results in the Journal of the American Statistical Association [90].

Monte Carlo methods are widely used in statistical simulations of physical systems. The core idea of Monte Carlo is to study a system using repeated random sampling to obtain numerical results. This approach is often the simplest way to solve a problem, and sometimes the only feasible way. The main drawback of Monte Carlo methods is that, depending on the system, they may require a very high number of steps to reach a reasonable convergence and, for this reason, they can be computationally expensive to carry out. With the continuous improvement of modern computers, this problem is getting progressively less relevant over time, but it is still of concern.

An important and well studied type of Monte Carlo technique is the Markov Chain Monte Carlo method (MCMC) [91]. In this work we will use a particular version of MCMC, called Gibbs sampling. Let us consider a system of interest described by several random variables $A, B, C, \ldots$ and let us say we are interested in simulating the evolution of the system, sampling from the joint distribution $P(A, B, C, \ldots)$. This distribution is often complicated and difficult to calculate, if not impossible. The main idea of Gibbs sampling is to sample one variable at the time, while keeping the others fixed. At every step thus only one variable of the system is varied and the sequence obtained by such evolution is forming 
a Markov chain. It can be demonstrated that, under particular conditions and once the Markov chain has reached equilibrium, sampling from the Markov chain is equivalent as sampling from a probability distribution called the equilibrium distribution. Designing an MCMC properly, it is thus possible to sample from a particular probability distribution of interest. More details on Markov chains and MCMC algorithms can be found in [91].

\subsection{The Kinetic Monte Carlo Algorithm}

The Kinetic Monte Carlo algorithm (KMC) is a variant of Monte Carlo intended to simulate the time evolution (hence the term kinetic) of a particular process of interest. In this thesis, as KMC we mean a particular variant of Gibbs sampler. We will explain in details this algorithm in the following of the present section.

A particular molecular orbital level in the system is specified with an upper case letter $M=(i, \alpha)$, identifying the orbital level $\alpha$ of the molecule $i$. We will imply in the notation that the orbital $M$ belongs to the molecule $i$ while the orbital $N$ belongs to the molecule $j$ (where $i$ and $j$ are different molecules). The flowchart of the algorithm is presented in figure 4.1. The initial condition (point 1) is composed by the MD simulated structure and by the initial position of the charges involved in the simulation. At a given time, every charge carrier hops from one molecular level $M$ to another molecular level $N$ with a rate given by the Marcus formula (section 2.1)

$$
w_{M N}=\frac{2 \pi}{\hbar}\left|H_{N M}\right|^{2} \sqrt{\frac{1}{4 \pi k_{B} T \lambda_{N M}}} \exp \left(-\frac{\left(-q \vec{E} \cdot \Delta \vec{r}_{N M}+\Delta E_{N M}+\lambda_{N M}\right)^{2}}{4 \lambda_{N M} k_{B} T}\right),
$$

$H_{N M}$ is the transfer integral expressing the electronic coupling of the levels $M$ and $N, \Delta E_{N M}$ is the energy difference between their two ground vibrational states and $\lambda_{N M}$ is the reorganization energy required for all the structural adjustments of the system after the hopping has happened. For the charge carrier on the orbital $M$, we calculate the rates $w_{M N}$ for every possible acceptor orbital $N$ within a given cut-off (point 2). The total escape rate for the charge on the orbital $M$ is thus $R_{M}=\sum_{N} w_{M N}$. In the general case of multiple charge carriers, we can think of every charge carrier $a$ sitting on the orbital $M_{a}$ and having a total escape rate $R_{M_{a}}$ (point 3). The evolution of the system is determined thanks to a weighted random selection algorithm (WRS), where the probability of selecting a particular value depends on its associated weight. First the hopping charge carrier is selected using as weights the total escape rates $R_{M_{a}}$. This select the charge $c$ that is jumping, thus identifying the origin of the hop (point 4). Let us assume that the hopping charge $c$ is sitting on the orbital $M$. The particular hop to perform is selected with another WRS using as weights the $w_{M N}$. In this way we select the destination of the hop (point 5). The time $\Delta t$ after which this hop occurs is extracted from an exponential distribution $\sim R_{M} \exp \left(-R_{M} \Delta t\right)$, where $R_{M}$ is the total escape rate from the orbital $M, R_{M}=\sum_{N} w_{M N}$ (point 6). Now the hop is performed and the time is updated (point 7). Several stopping criteria can be specified. A stopping criterion can be the simulation time, the number of hops, but also some positional 


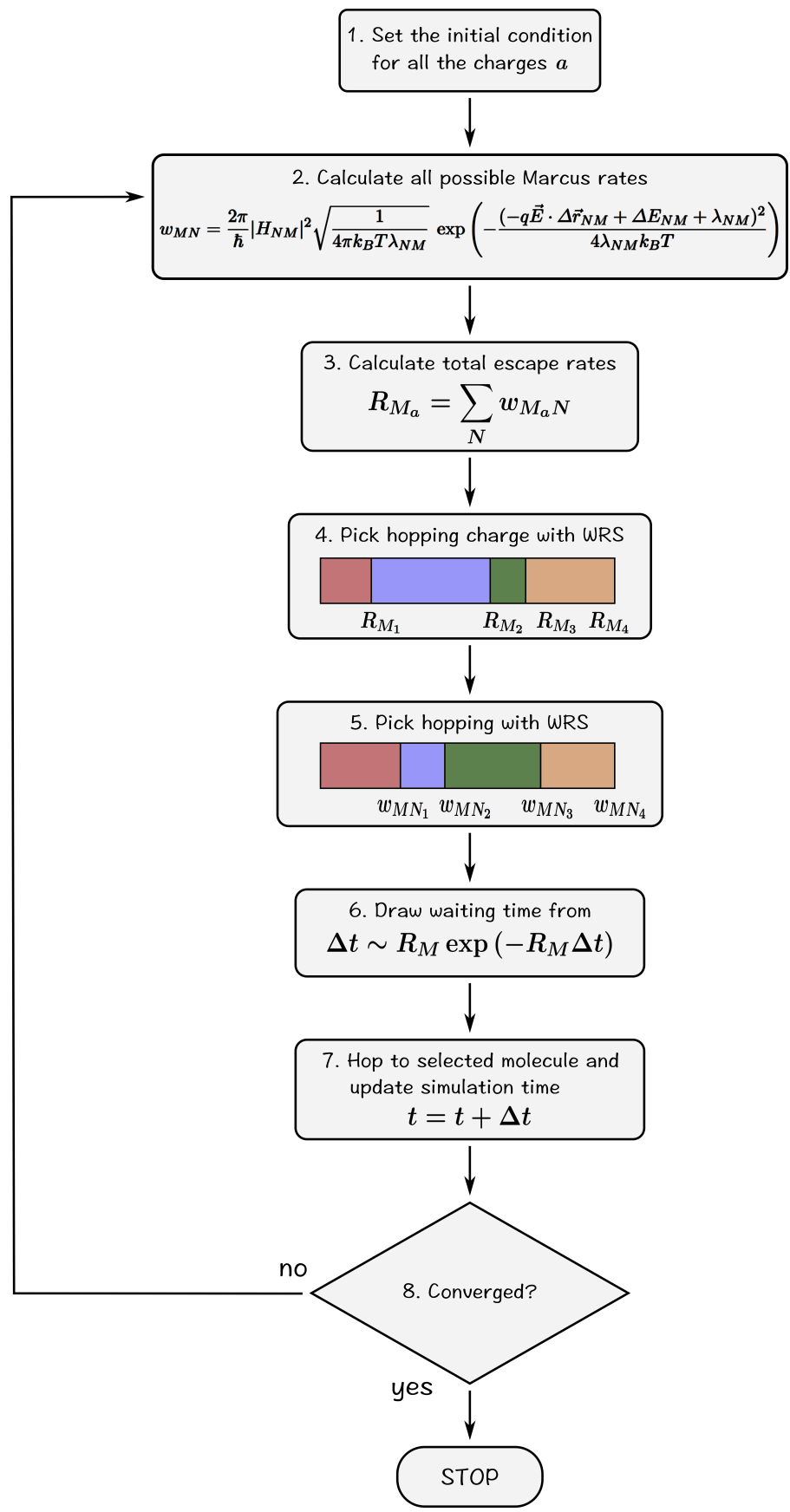

Figure 4.1. Flowchart of the KMC simulation used in this thesis. 
criteria like the charge carrier passing through a particular plane in space. If no stopping criterion is respected (point 8), we go back at point 2 and continue the simulation. 


\section{CHAPTER 5}

\section{Conclusions and Outlook}

The work included in this Dissertation contributed to the current theoretical investigations on the functioning of organic solar cells. We proceeded with a bottom up approach, first focusing on the charge transport in the single phase (a single organic material) and subsequently shifting our attention on the interface between two organic materials. The atomistic model employed allows us to describe in detail the electrostatic interactions happening in organic materials between a charge carrier and the nearby molecules. In a continuous iterative process, the model has been progressively tested and refined till the present version. KMC simulations have been a useful tool for testing the theory against experimental data and supporting the analytical reasoning. The results and a brief description of the work will be presented in this chapter.

\subsection{Mobility in the Single Phase}

The correlation of the energy landscape is one of the main determinant factors for conduction [64]. One of the first pioneering works on the simulations of charge transport in organic materials was published by Bässler [35]. These simulations were based on a crystalline lattice using a Miller-Abrahams hopping rate [46]. The disorder of the electronic levels (LUMO for electrons and HOMO for holes) of the system had been obtained by considering all the molecular energy levels independently and identically distributed (i.i.d.) with gaussian random variables. This approach allowed to gain great insight into the charge transport in organic materials and to achieve qualitatively the correct mobility temperature dependence, but could not obtain Poole-Frenkel type of mobility field dependence [35]. Introducing a realistic spatial disorder in the bulk, combined with DOD transfer integrals (Section 3.2), again allowed for a temperature dependence of mobility in 
agreement with experiments, but could not recover the expected mobility field dependence. [44] This problem has been studied deeper and solved in Paper I-II. The correlation coming from randomly oriented dipoles (randomly oriented molecules in an amorphous material) derived by Novikov and Vannikov [75, 76], allows to recover the Poole-Frenkel formula [92] in what is called the correlation region [64]. Inspired by these works we computed the energy shift of the HOMO level of every molecule in the box according to the dipoles of the neighbouring molecules. The dipole correlation allowed us to retrieve the expected Poole-Frenkel dependency for the hole mobility in $4 \mathrm{PV}$ and also to identify the transition fields separating the 3 regions of interest, allowing us to estimate the position of these regions on the field axis, see Paper I. The development of this line of thinking led to the efficiency diagram explained in Section 2.1 and used in Paper III. The DOS alone is not sufficient information to predict the conduction in a material but the correlation is equally important. The drawback of Paper I is that we had to introduce a scaling factor to have a significant effect on mobility, since the dipoles of neutral 4PV were too small to affect conduction otherwise. In Paper II, the treatment of the environment improved since we include atomic charges and polarization interactions. We focus here on $\mathrm{C}_{60}$, a simple well studied material for which experimental mobility field dependence and mobility temperature dependence are available $[93,94] . \mathrm{C}_{60}$ is a spherical fullerene molecule, presenting no electrostatic permanent dipoles or multipoles in its neutral state. Due to this fact, it was not clear what would produce the broadening of the DOS and the respective correlation of the energies. When neglecting polarization of the environment, poor results are indeed obtained in terms of both mobility field and temperature dependence, almost no dependence is observed in correlation region. Activating polarization effects as induced dipoles on the nearby molecules, lead to an energy correction for the single site energies and for the external reorganization happening after each hopping. In particular $\mathrm{C}_{60}$ is a highly polarizable molecule and the external reorganization energy revealed to be the most important factor, shifting the inverse Marcus region to higher fields and allowing a good qualitative agreement with experiments for both mobility field and temperature dependence. In Paper III, the situation got a bit more complicated, with the study of mobility in $\mathrm{PC}_{61} \mathrm{BM}$. It is interesting to notice how the $\mathrm{O}$ energetic contribution showed an asymmetric distribution due to the oxygen in the carbonyl group interacting with the $\mathrm{C}_{60}$ cage. The $\mathrm{C}$ contribution is very strong in this molecule and it is the dominant one, leading the OCP contribution with a standard deviation of about $0.25 \mathrm{eV}$. In this material then also the DOS has a strong impact on the mobility field dependence. $\mathrm{PC}_{61} \mathrm{BM}$ is also a highly polarizable molecule, thus both DOS and external reorganization energy have a great influence on the mobility. In Paper IV, we have investigated how the peripheral substitution of discotic molecules can affect the transport properties. In particular we were able to establish relationship between the deformation parameters of the stack (rise, twist and shift) and the distribution of transfer integrals. Our results were found in excellent agreement with the experiment. 


\subsection{CT state splitting at the Interface}

After having studied mobility in the single phase, we proceed to study the dynamics of multiple charges. In particular we focus on the CT state splitting (Paper V and $\mathrm{VI}$ ), that is intimately related to the efficient generation of free charge carriers in a solar cell (Chapter 1). The CT state splitting diagram introduced in Section 2.2 has been used to analyze two interfaces: $\mathrm{C}_{60}$ /anthracene (Paper $\mathrm{V}$ ) and $\mathrm{PC}_{71} \mathrm{BM} / 2$ TQ1 (Paper VI). In both papers we found out how the CT state diagram enables to predict qualitative behaviours of the $\mathrm{CT}$ state splitting quite well, compared with the following KMC simulations. When one of the two charges composing the CT state moves, the Coulombic interactions binding the CT state decrease, and if the electric field is too high one of the two charges might be in deep inverse Marcus region and thus not able move to the electrode. This fact usually prevents the full splitting of the CT state, as observed in Paper V. In particular we also saw how the relative orientation of the anthracene at the interface with $\mathrm{C}_{60}$ affects the CT state splitting and how the cause of this dependence can be easily understood with the CT state splitting diagram. In Paper VI we could also rationalize the qualitative behaviour of the $\mathrm{CT}$ splitting and charge collection average times in the KMC simulations.

It is expected in the literature that interfacial dipoles play an important role in helping the charge splitting at the interface [95-98]. This effect is reflected on the $\Delta E_{N M}$, the polarization at the interface helps to push away the charges that are more stabilized in the bulk (as also observed in Paper V and VI). Another effect of polarization is on the reorganization energy, that leads to shorter and wider gaussians (given the dependency of Marcus rate on $\lambda$ ). This favours the overlapping between CT splitting and free conduction gaussians thus favouring charge collection.

In conclusion it is possible through the CT state splitting diagram to understand if two materials are suitable to be used together in an organic solar cell (Paper VI and [65]). The requirements are that the two materials have similar energetic barriers at the interface $\Delta E_{N M}$, this will favour the overlap between electrons and holes CT splitting gaussians. To have a good overlap between CT state splitting and free conduction gaussians, instead the energetic barrier $\Delta E_{N M}$ should be small compared to the gaussians standard deviations. For the external reorganization energy a balance has to be found, since it helps the gaussians overlap, but at the same time materials with an excessively strong external reorganization energy will have very low rates and thus poor transport properties.

\subsection{Future Perspectives}

The work presented in this Dissertation helps to shine light on the main pitfalls of the actual theory. Innovative ways to calculate environmental effect and to analyze charge transport in organic materials have been presented. Still there are two main open questions on which future effort should be concentrated for a deep understanding of the physics of organic solar cells. 
First of all the fields needed in our KMC simulations to obtain conduction in the single phase are very high compared with experiments (Papers I, II and III). A cause for so high electric fields could be due to the fact that in our model the transfer integrals are considered independent on the electric field value, as detailed in Chapter 3. This fact (as already discussed in Section 2.1) is supported in literature [62], nevertheless this study uses calculations at the ZINDO level without extended basis functions, thus neglecting relevant polarization effects that might change the conclusions. Moreover, it is possible that Marcus theory might not be enough to describe charge transport in organic materials and Marcus-LevichJortner theory might be more appropriate, allowing tunneling effects across the potential barrier and lowering the strength of the electric fields needed. Some works in the literature are advancing in this direction [60] and we also think a more detailed study on this topic is necessary. In Paper II indeed we demonstrate that at experimental fields our simulations are in the percolation region and we show how this is a fact that depends on the model used, it is not possible to solve this problem adjusting parameters (even if fitting parameters was not the scope of the paper, this represents a proof of principle). In Paper III this conviction became even stronger, employing the efficiency diagram and noticing discrepancies with the experimental mobility temperature dependencies.

Also the CT state splitting inherits this drawback (Paper V and VI). But even assuming that Marcus Theory is enough for the modelling of organic systems, we arrive to the second open problem, the inclusion of excited molecular orbital levels in the model. The hot CT state definitely needs to be considered, allowing extra paths in the CT state dissociation. An analysis based on the CT state dissociation would save computational times of the KMC and increase our understanding. Thus a development of this theory for hot CT states is desirable, taking into account the competing rates of relaxation among different levels. 
[1] Mulvaney, D.; Bolam, V.; Cendejas, M.; Davis, S.; Ornelas, L.; Kim, S.; Mau, S.; Rowan, W.; Sanz, E.; Satre, P.; Sridhar, A.; Young, D.; MacDonald, K. Toward a Just and Sustainable Solar Energy Industry. Tech. rep., Silicon Valley Toxics Coalition, 2009.

[2] Forrest, S. R. The Path to Ubiquitous and Low-Cost Organic Electronic Appliances on Plastic. Nature 2004, 428, 911-918.

[3] Kim, J.-H.; Han, M. J.; Seo, S. Flexible, Stretchable, and Patchable Organic Devices Integrated on Freestanding Polymeric Substrates. J. Polym. Sci., Part B: Polym. Phys. 2015, 53, 453-460.

[4] Facchetti, A. $\pi$-Conjugated Polymers for Organic Electronics and Photovoltaic Cell Applications. Chem. Mater. 2011, 23, 733-758.

[5] Gundlach, D. J. Low Power, High Impact. Nat. Mater. 2007, 6, 173-174.

[6] Wohrle, D.; Dieter, M. Organic Solar Cells. Advanced Materials 1991, 3, 129.

[7] Shockley, W.; Queisser, H. J. Detailed Balance Limit of Efficiency of p-n Junction Solar Cells. Journal of Applied Physics 1961, 32, 510.

[8] Green, M. A. Third generation photovoltaics: Ultra-high conversion efficiency at low cost. Progress in Photovoltaics: Research and Applications 2001, 9, $123-135$.

[9] Xiao, S.; Xu, S. High-Efficiency Silicon Solar Cells-Materials and Devices Physics. Critical Reviews in Solid State and Materials Sciences 2014, 39, $277-317$. 
[10] Alharbi, F. H.; Kais, S. Theoretical limits of photovoltaics efficiency and possible improvements by intuitive approaches learned from photosynthesis and quantum coherence. Renewable and Sustainable Energy Reviews 2015 , 43, 1073-1089.

[11] Green, M. A.; Emery, K.; Hishikawa, Y.; Warta, W.; Dunlop, E. D. Solar cell efficiency tables (Version 48). Progress in Photovoltaics: Research and Applications 2016, 24, 905-913.

[12] NREL Best Research-Cell Efficiencies. http://www.nrel.gov/pv/assets/ images/efficiency_chart.jpg, accessed: 2016-12-02.

[13] Cheng, P.; Zhan, X. Stability of organic solar cells: challenges and strategies. Chem. Soc. Rev. 2016, 45, 2544-2582.

[14] Jørgensen, M.; Norrman, K.; Krebs, F. C.; Jorgensen, M.; Norrman, K.; Krebs, F. C. Stability/Degradation of Polymer Solar Cells. Sol. Energy Mater. Sol. Cells 2008, 92, 686-714.

[15] Locci, S. Modeling of Physical and Electrical Characteristics of Organic Thin Film Transistors. Ph.D. thesis, University of Cagliari, 2009.

[16] Aulin, Y. V. Excitons in Organic Semiconductors. Master's thesis, University of Groningen, 2010.

[17] Vandewal, K.; Tvingestedt, K.; Inganäs, O. Charge Transfer States in Organic Donor-Acceptor Solar Cells. Semicond. Semimetals 2011, 85, 261-295.

[18] Zhu, X.; Yang, Q.; Muntwiler, M. Charge-Transfer Excitons at Organic Semiconductor Surfaces and Interfaces. Acc. Chem. Res. 2009, 42, 17791787.

[19] Lee, J.; Vandewal, K.; Yost, S. R.; Bahlke, M. E.; Goris, L.; Baldo, M. A.; Manca, J. V.; Voorhis, T. V. Charge Transfer State Versus Hot Exciton Dissociation in Polymer-Fullerene Blended Solar Cells. J. Am. Chem. Soc. 2010, 132, 11878-11880.

[20] Brabec, C. J.; Hauch, J. A.; Schilinsky, P.; Waldauf, C. Production Aspects of Organic Photovoltaics and Their Impact on the Commercialization of Devices. MRS Bull. 2005, 30, 50-52.

[21] Brédas, J.-L.; Norton, J. E.; Cornil, J.; Coropceanu, V. Molecular Understanding of Organic Solar Cells: The Challenges. Acc. Chem. Res. 2009, 42, 1691-1699.

[22] von Hauff, E. The Role of Molecular Structure and Conformation in Polymer Electronics. Semiconductors and Semimetals 2011, 85, 231-260.

[23] Deibel, C. Photocurrent Generation in Organic Solar Cells. Semiconductors and Semimetals 2011, 85, 297-330. 
[24] Lunt, R. R.; Giebink, N. C.; Belak, A.; Benziger, J. B.; Forrest, S. R. Exciton diffusion lengths of organic semiconductor thin films measured by spectrally resolved photoluminescence quenching. J. Appl. Phys. 2009, 105, 053711.

[25] Buxton, G. A.; Clarke, N. Computer Simulation of Polymer Solar Cells. Modell. Simul. Mater. Sci. Eng. 2007, 15, 13-26.

[26] Mirjani, F.; Renaud, N.; Gorczak, N.; Grozema, F. C. Theoretical Investigation of Singlet Fission in Molecular Dimers: The Role of Charge Transfer States and Quantum Interference. The journal of physical chemistry C 2014, $118,14192$.

[27] Tao, Y.; Yuan, K.; Chen, T.; Xu, P.; Li, H.; Chen, R.; Zheng, C.; Zhang, L.; Huang, W. Thermally Activated Delayed Fluorescence Materials Towards the Breakthrough of Organoelectronics. Advanced materials 2014, 1-28.

[28] Hannewald, K.; Stojanović, V.; Schellekens, J.; Bobbert, P.; Kresse, G.; Hafner, J. Theory of polaron bandwidth narrowing in organic molecular crystals. Physical Review B 2004, 69, 075211.

[29] Hannewald, K.; Bobbert, P. A. Ab initio theory of charge-carrier conduction in ultrapure organic crystals. Applied Physics Letters 2004, 85, 1535.

[30] Ortmann, F.; Bechstedt, F.; Hannewald, K. Theory of charge transport in organic crystals: Beyond Holstein's small-polaron model. Physical Review B 2009, 79, 235206.

[31] Ortmann, F.; Bechstedt, F.; Hannewald, K. Charge transport in organic crystals: Theory and modelling. Physica Status Solidi (B) 2011, 248, 511525 .

[32] Koster, L. J. A.; Smits, E. C. P.; Mihailetchi, V. D.; Blom, P. W. M. Device model for the operation of polymer/fullerene bulk heterojunction solar cells. Physical Review B 2005, 72, 085205.

[33] Gruber, M.; Stickler, B.; Trimmel, G.; Schürrer, F.; Zojer, K. Impact of Energy Alignment and Morphology on the Efficiency in Inorganic--Organic Hybrid Solar Cells. Org. Electron. 2010, 11, 1999-2011.

[34] de Falco, C.; Porro, M.; Sacco, R.; Verri, M. Multiscale modeling and simulation of organic solar cells. Computer Methods in Applied Mechanics and Engineering 2012, 245-246, 102-116.

[35] Bässler, H. Charge Transport in Disordered Organic Photoconductors. A Monte Carlo Study. Phys. Status Solidi B 1993, 175, 15.

[36] Watkins, P. K.; Walker, A. B.; Verschoor, G. L. B. Dynamical Monte Carlo Modelling of Organic Solar Cells: The Dependence of Internal Quantum Efficiency on Morphology. Nano lett. 2005, 5, 1814. 
[37] Kirkpatrick, J.; Marcon, V.; Nelson, J.; Kremer, K.; Andrienko, D. Charge Mobility of Discotic Mesophases: A Multiscale Quantum and Classical Study. Phys. Rev. Lett. 2007, 98, 227402.

[38] Olivier, Y.; Muccioli, L.; Lemaur, V.; Geerts, Y. H.; Zannoni, C.; Cornil, J. Theoretical Characterization of the Structural and Hole Transport Dynamics in Liquid-Crystalline Phthalocyanine Stacks. J. Phys. Chem. B 2009, 113, $14102-14111$.

[39] Idé, J.; Méreau, R.; Ducasse, L.; Castet, F. Supramolecular Organization and Charge Transport Properties of Self-Assembled $\pi-\pi$ Stacks of Perylene Diimide Dyes. J. Phys. Chem. B 2011, 115, 5593-5603.

[40] Poelking, C.; Cho, E.; Malafeev, A.; Ivanov, V.; Kremer, K.; Risko, C.; Brédas, J. L.; Andrienko, D. Characterization of Charge-Carrier Transport in Semicrystalline Polymers: Electronic Couplings, Site Energies, and ChargeCarrier Dynamics in Poly(Bithiophene-Alt-Thienothiophene) [PBTTT]. J. Phys. Chem. C 2013, 117, 1633-1640.

[41] Deibel, C.; Strobel, T.; Dyakonov, V. Origin of the Efficient Polaron-Pair Dissociation in Polymer-Fullerene Blends. Phys. Rev. Lett. 2009, 103, 036402.

[42] Vehoff, T.; Baumeier, B.; Troisi, A.; Andrienko, D. Charge Transport in Organic Crystals: Role of Disorder and Topological Connectivity. J. Am. Chem. Soc. 2010, 132, 11702-11708.

[43] Rühle, V.; Kirkpatrick, J.; Andrienko, D. A Multiscale Description of Charge Transport in Conjugated Oligomers. J. Chem. Phys. 2010, 132, 134103.

[44] Jakobsson, M.; Linares, M.; Stafström, S. Monte Carlo Simulations of Charge Transport in Organic Systems With True Off-Diagonal Disorder. J. Chem. Phys. 2012, 137, 114901.

[45] Volpi, R.; Kottravel, S.; Steen Nørby, M.; Stafström, S.; Linares, M. Effect of Polarization on the Mobility of $\mathrm{C}_{60}$ : A Kinetic Monte Carlo Study. J. Chem. Theory Comput. 2016, 12, 812-824.

[46] Miller, A.; Abrahams, E. Impurity Conduction at Low Concentrations. Phys. Rev. 1960, 120, 745.

[47] Marcus, R. A. On the Theory of Oxidation-Reduction Reactions Involving Electron Transfer. I. J. Chem. Phys. 1956, 24, 966.

[48] Heiber, M. C.; Dhinojwala, A. Efficient Generation of Model Bulk Heterojunction Morphologies for Organic Photovoltaic Device Modeling. Phys. Rev. Appl. 2014, 2, 014008.

[49] Mikhnenko, O. V.; Blom, P. W. M.; Nguyen, T.-Q. Exciton diffusion in organic semiconductors. Energy Environ. Sci. 2015, 8, 1867-1888. 
[50] Barford, W.; Tozer, O. R. Theory of exciton transfer and diffusion in conjugated polymers. Journal of Chemical Physics 2014, 141.

[51] Tapping, P. C.; Clafton, S. N.; Schwarz, K. N.; Kee, T. W.; Huang, D. M. Molecular-Level Details of Morphology-Dependent Exciton Migration in Poly(3-hexylthiophene) Nanostructures. Journal of Physical Chemistry $C$ 2015, 119, 7047-7059.

[52] Yi, Y.; Coropceanu, V.; Brédas, J.-L. Exciton Dissociation and ChargeRecombination Processes in Pentacene/C60: Theoretical Insight into the Impact of Interface Geometry. Journal of the American Chemical Society 2009, 131, 15777-15783.

[53] Yi, Y.; Coropceanu, V.; Brédas, J.-L. A comparative theoretical study of exciton-dissociation and charge-recombination processes in oligothiophene/fullerene and oligothiophene/perylenediimide complexes for organic solar cells. Journal of Materials Chemistry 2011, 21, 1479.

[54] van Eersel, H.; Janssen, R. A. J.; Kemerink, M. Mechanism for efficient photoinduced charge separation at disordered organic heterointerfaces. Advanced Functional Materials 2012, 22, 2700-2708.

[55] Caruso, D.; Troisi, A. Long-range exciton dissociation in organic solar cells. Proceedings of the National Academy of Sciences 2012, 109, 13498.

[56] Fu, Y. T.; Da Silva Filho, D. A.; Sini, G.; Asiri, A. M.; Aziz, S. G.; Risko, C.; Brédas, J. L. Structure and disorder in squaraine-C60 organic solar cells: A theoretical description of molecular packing and electronic coupling at the donor-acceptor interface. Advanced Functional Materials 2014, 24, $3790-3798$.

[57] Levich, V.; Dogonadze, R. Theory of non-radiation electron transitions from ion to ion in solutions. Dok. Akad. Nauk SSSR 1959, 124, 123.

[58] Levich, V. Present state of the theory of oxidation-reduction in solution (bulk and electrode reactions). Adv. Electrochem. Electrochem. Eng. 1966, 4, 249.

[59] Jortner, J. Temperature dependent activation energy for electron transfer between biological molecules. J. Chem. Phys. 1976, 64, 4860.

[60] Asadi, K.; Kronemeijer, A. J.; Cramer, T.; Jan Anton Koster, L.; Blom, P. W. M.; de Leeuw, D. M. Polaron hopping mediated by nuclear tunnelling in semiconducting polymers at high carrier density. Nature Communications 2013, 4, 1710 .

[61] Sancho-García, J. C.; Horowitz, G.; Brédas, J. L.; Cornil, J. Effect of an External Electric Field on the Charge Transport Parameters in Organic Molecular Semiconductors. J. Chem. Phys. 2003, 119, 12563-12568. 
[62] Olivier, Y.; Lemaur, V.; Brédas, J. L.; Cornil, J. Charge Hopping in Organic Semiconductors: Influence of Molecular Parameters on Macroscopic Mobilities in Model One-Dimensional Stacks. J. Phys. Chem. A 2006, 110, 6356-6364.

[63] Volpi, R.; Nassau, R.; Nørby, M. S.; Linares, M. Theoretical study of the charge-transfer state separation within Marcus Theory: the C60-anthracene case study. Applied Materials \& Interfaces 2016, 8, 24722.

[64] Dunlap, D. H.; Kenkre, V. M.; Parris, P. E. What is behind the $\sqrt{E}$ ? Journal of Imaging Science and Technology 1999, 43, 437.

[65] Volpi, R.; Linares, M. Organic Solar Cells. Specialist Periodic Reports Chemical Modelling, RSC 2016, 13, 1-26.

[66] McMahon, D. P.; Cheung, D. L.; Troisi, A. Why Holes and Electrons Separate So Well in Polymer/Fullerene Photovoltaic Cells. J. Phys. Chem. Lett. 2011, $2,2737-2741$.

[67] Jailaubekov, A. E.; Willard, A. P.; Tritsch, J. R.; Chan, W.-L.; Sai, N.; Gearba, R.; Kaake, L. G.; Williams, K. J.; Leung, K.; Rossky, P. J.; Zhu, X.Y. Hot Charge-Transfer Excitons Set the Time Limit for Charge Separation at Donor/Acceptor Interfaces in Organic Photovoltaics. Nature materials 2013, 12, 66-73.

[68] Grancini, G.; Maiuri, M.; Fazzi, D.; Petrozza, a.; Egelhaaf, H.-J.; Brida, D.; Cerullo, G.; Lanzani, G. Hot Exciton Dissociation in Polymer Solar Cells. Nat. Mater. 2013, 12, 29-33.

[69] Gélinas, S.; Rao, A.; Kumar, A.; Smith, S. L.; Chin, A. W.; Clark, J.; Poll, T. S. V. D.; Bazan, G. C.; Friend, R. H. Ultrafast Long-Range Charge Separation in Organic Semiconductor Photovoltaic Diodes. Science 2014, 343,512 .

[70] Smith, S. L.; Chin, A. W. Ultrafast Charge Separation and Nongeminate Electron-Hole Recombination in Organic Photovoltaics. Phys. Chem. Chem. Phys. 2014, 16, 20305-20309.

[71] Melianas, A.; Etzold, F.; Savenije, T. J.; Laquai, F.; Inganäs, O.; Kemerink, M. Photo-Generated Carriers Lose Energy During Extraction From PolymerFullerene Solar Cells. Nat. Commun. 2015, 6, 8778.

[72] D’Avino, G.; Muccioli, L.; Olivier, Y.; Beljonne, D. Charge Separation and Recombination at Polymer-Fullerene Heterojunctions: Delocalization and Hybridization Effects. J. Phys. Chem. Lett. 2016, 7, 536-540.

[73] van der Hofstad, T. G. J.; Di Nuzzo, D.; van den Berg, M.; Janssen, R. A. J.; Meskers, S. C. J. Influence of Photon Excess Energy on Charge Carrier Dynamics in a Polymer-Fullerene Solar Cell. Adv. Energy Mater. 2012, 2, 1095-1099. 
[74] Gao, F.; Inganäs, O. Charge Generation in Polymer-Fullerene BulkHeterojunction Solar Cells. Phys. Chem. Chem. Phys. 2014, 16, 20291.

[75] Novikov, S.; A., V. Distribution of electrostatic potential in a lattice of randomly oriented dipoles. Journal of Experimental and Theoretical Physics 1994, 79, 482 .

[76] Novikov, S.; A., V. Cluster Structure in the Distribution of the Electrostatic Potential in a Lattice of Randomly Oriented Dipoles. Journal of Physical Chemistry 1995, 99, 14573.

[77] Cornil, J.; Beljonne, D.; Calbert, J.-P.; Brédas, J.-L. Interchain Interactions in Organic Pi-Conjugated Materials : Impact on Electronic Structure, Optical Response, and Charge Transport. Adv. Mater. 2001, 13, 1053-1067.

[78] Valeev, E. F.; Coropceanu, V.; da Silva Filho, D. A.; Salman, S.; Brédas, J.-L. Effect of Electronic Polarization on Charge-Transport Parameters in Molecular Organic Semiconductors. J. Am. Chem. Soc. 2006, 128, 9882.

[79] Mulliken, R.; Rieke, C.; Orloff, D.; Orloff, H. Formulas and Numerical Tables for Overlap Integrals. Journal of Chemical Physics 1949, 17, 1248.

[80] Hansson, A.; Stafström, S. Intershell conductance in multiwall carbon nanotubes. Physical Review B 2003, 67, 075406.

[81] Kirkpatrick, J. An Approximate Method for Calculating Transfer Integrals Based on the ZINDO Hamiltonian. Int. J. Quantum Chem. 2008, 108, 51-56.

[82] Baumeier, B.; Kirkpatrick, J.; Andrienko, D. Density-functional based determination of intermolecular charge transfer properties for large-scale morphologies. Phys. Chem. Chem. Phys. 2010, 12, 11103-13.

[83] Petelenz, P. Polarization Effects in Molecular Crystals. Chemical Physics Letter 1984, 103, 369-372.

[84] Bussac, M. N.; Picon, J. D.; Zuppiroli, L. The impact of molecular polarization on the electronic properties of molecular semiconductors. Europhysics Letters 2004, 66, 392-398.

[85] Castet, F.; Aurel, P.; Fritsch, A.; Ducasse, L.; Liotard, D.; Linares, M.; Cornil, J.; Beljonne, D. Electronic Polarization Effects on Charge Carriers in Anthracene: A Valence Bond Study. Phys. Rev. B 2008, 77, 115210.

[86] Brédas, J.-L.; Beljonne, D.; Coropceanu, V.; Cornil, J. Charge-Transfer and Energy-Transfer Processes in $\pi$-Conjugated Oligomers and Polymers: A Molecular Picture. Chem. Rev. 2004, 104, 4971-5003.

[87] Rühle, V.; Lukyanov, A.; May, F.; Schrader, M.; Vehoff, T.; Kirkpatrick, J.; Baumeier, B.; Andrienko, D. Microscopic Simulations of Charge Transport in Disordered Organic Semiconductors. Journal of Chemical Theory and Computation 2011, 7, 3335. 
[88] Monte Carlo method. Wikipedia: The free encyclopedia http://en.wikipedia.org/wiki/Monte_Carlo_method, accessed: 2015-09-08.

[89] Metropolis, N. The Beginning of the Monte Carlo Method. Los Alamos Science 1987, 15, 125.

[90] Metropolis, N.; Ulam, S. The Monte Carlo Method. Journal of the American Statistical Association 1949, 44, 335.

[91] Andrieu, C.; Doucet, A.; Holenstein, R. Particle Markov chain Monte Carlo methods. Journal of the Royal Statistical Society: Series B (Statistical Methodology) 2010, 72, 269-342.

[92] Frenkel, J. On Pre-Breakdown Phenomena in Insulators and Electronic Semi-Conductors. Physical Review 1938, 54, 647.

[93] Fishchuk, I. I.; Kadashchuk, A.; Novikov, S. V.; Ullah, M.; Genoe, J.; Sariciftci, N. S.; Sitter, H.; Bässler, H. Origin of Electric Field Dependence of the Charge Mobility and Spatial Energy Correlations in C60-Based Field Effect Transistors. Molecular Crystals and Liquid Crystals 2014, 589, 18-28.

[94] Pivrikas, A.; Ullah, M.; Simbrunner, C.; Sitter, H.; Neugebauer, H.; Serdar Sariciftci, N. Comparative study of bulk and interface transport in disordered fullerene films. Physica Status Solidi (B) Basic Research 2011, 248, 2656.

[95] Arkhipov, V. I.; Heremans, P.; Bässler, H. Why is exciton dissociation so efficient at the interface between a conjugated polymer and an electron acceptor? Applied Physics Letters 2003, 82, 4605.

[96] Nenashev, A. V.; Wiemer, M.; Jansson, F.; Baranovskii, S. D. Theory to exciton dissociation at the interface between a conjugated polymer and an electron acceptor. Journal of Non-Crystalline Solids 2012, 358, 2508-2511.

[97] Verlaak, S.; Beljonne, D.; Cheyns, D.; Rolin, C.; Linares, M.; Castet, F.; Cornil, J.; Heremans, P. Electronic Structure and Geminate Pair Energetics at Organic-Organic Interfaces: The Case of Pentacene/ $\mathrm{C}_{60}$ Heterojunctions. Advanced Functional Materials 2009, 19, 3809-3814.

[98] Cornil, J.; Verlaak, S.; Martinelli, N.; Mityashin, A.; Olivier, Y.; Van Regemorter, T.; D’Avino, G.; Muccioli, L.; Zannoni, C.; Castet, F.; Beljonne, D.; Heremans, P. Exploring the energy landscape of the charge transport levels in organic semiconductors at the molecular scale. Accounts of Chemical Research 2013, 46, 434-443.

[99] Abraham, M. J.; van der Spoel, D.; Lindahl, E.; Hess, B.; the GROMACS development team GROMACS User Manual version 5.0.7. www.gromacs.org, 2015.

[100] Parker, E. N. Tensor Virial Equations. Physical Review 1954, 96, 1686.

[101] Jensen, F. Introduction to Computational Chemistry. Wiley, 2007. 
[102] Jorgensen, W. L.; Tirado-Rives, J. The OPLS Potential Functions for Proteins. Energy Minimizations for Crystals of Cyclic Peptides and Crambin. J. Am. Chem. Soc. 1988, 110, 1657-1666.

[103] Jorgensen, W. L.; Maxwell, D. S.; Tirado-Rives, J. Development and Testing of the OPLS All-Atom Force Field on Conformational Energetics and Properties of Organic Liquids. J. Am. Chem. Soc. 1996, 118, 11225-11236.

[104] Berendsen, H. J. C.; van der Spoel, D.; van Drunen, R. GROMACS : A Message-Passing Parallel Molecular Dynamics Implementation. Comput. Phys. Commun. 1995, 91, 43-56.

[105] Lindahl, E.; Hess, B.; van der Spoel, D. GROMACS 3.0 : A Package for Molecular Simulation and Trajectory Analysis. J. Mol. Model. 2001, 7, $306-317$.

[106] van der Spoel, D.; Lindahl, E.; Hess, B.; Groenhof, G.; Mark, A. E.; Berendsen, H. J. C. GROMACS: Fast, Flexible, and Free. J. Comput. Chem. 2005, 26,1701 .

[107] Hess, B.; Kutzner, C.; van der Spoel, D.; Lindahl, E. GROMACS 4: Algorithms for Highly Efficient, Load-Balanced, and Scalable Molecular Simulation. J. Chem. Theory Comput. 2008, 4, 435-447.

[108] Rühle, V. Berendsen and Nose-Hoover thermostats. http://www2.mpipmainz.mpg.de/ andrienk/journal_club/thermostats.pdf, 2008, accessed: 2015-09-08.

[109] Hoover, W. G. Canonical dynamics: Equilibrium phase-space distributions. Phys. Rev. A 1985, 31, 1695.

[110] Andersen, H. C. Molecular dynamics simulations at constant pressure and/or temperature. The Journal of Chemical Physics 1980, 72, 2384.

[111] Nosé, S. A molecular dynamics method for simulations in the canonical ensemble. Mol. Phys. 2002, 100, 191.

[112] Parrinello, M.; Rahman, A. Polymorphic Transitions in Single Crystals: a New Molecular Dynamics Method. J. Appl. Phys. 1981, 52, 7182.

[113] Ising model. Wikipedia: The free encyclopedia http://en.wikipedia.org/wiki/Ising_model, accessed: 2015-09-08.

[114] Cai, W. ME346A Introduction to Statistical Mechanics: Handout 12. Ising Model. Stanford University http://micro.stanford.edu/ caiwei/me334/ ME346A_Lecture_Notes_Win2012.pdf, accessed: 2015-10-20.

[115] Pople, J. A.; Santry, D. P.; Segal, G. A. Approximate Self-Consistent Molecular Orbital Theory. I. Invariant Procedures. The Journal of Chemical Physics 1965, 43, S129. 
[116] Pople, J. A.; Segal, G. A. Approximate Self-Consistent Molecular Orbital Theory. II. Calculations with Complete Neglect of Differential Overlap. The Journal of Chemical Physics 1965, 43, S136.

[117] Pople, J. A. Approximate Self-Consistent Molecular-Orbital Theory. V. Intermediate Neglect of Differential Overlap. The Journal of Chemical Physics 1967, 47, 2026.

[118] Zerner, M. C.; Loew, G. H.; Kirchner, R. F.; Mueller-Westerhoff, U. T. An Intermediate Neglect of Differential Overlap Technique for Spectroscopy of Transition-Metal Complexes. Ferrocene. J. Am. Chem. Soc. 1980, 102, $589-599$.

[119] Hohenberg, P.; Kohn, W. Inhomogeneous electron gas. Physical Review 1964, 136,864 .

[120] Kohn, W. Nobel Lecture: Electronic structure of matter-wave functions and density functionals. Rev. Mod. Phys. 1999, 71, 1253-1266.

[121] Kohn, W.; Sham, L. J. Self-Consistent Equations Including Exchange and Correlation Effects. Physical Review B 1965, 140. 


\section{List of included Publications}

[I] Transition fields in organic materials: From percolation to inverted Marcus regime. A consistent Monte Carlo simulation in disordered PPV

R. Volpi, S. Stafström, M. Linares

Journal of Chemical Physics 2015, 142, 094503

[II] Effect of Polarization on the Mobility of $\mathrm{C}_{60}$ : A Kinetic Monte-Carlo Study

R. Volpi, S. Kottravel, M. Pedersen, S. Stafström, M. Linares

Journal of Chemical Theory and Computation 2016, 12, 812

[III] Mobility Field and Mobility Temperature Dependence in $\mathbf{P C}_{61} \mathbf{B M}$ L. Sousa, R. Volpi, D. A. da Silva Filho, M. Linares Submitted

[IV] Modeling Charge Transport of Discotic Liquid-Crystalline Triindoles: The Role of Peripheral Substitution

R. Volpi, A. C. Santos Camilo, D. A. da Silva Filho, J. T. López Navarrete, B. Gómez-Lor, M. C. Ruiz Delgado, M. Linares

Submitted

[V] Theoretical Study of the Charge-Transfer State Separation within Marcus Theory: the C60-Anthracene Case Study

R. Volpi, R. Nassau, M. Steen Nørby, M. Linares

Applied Materials and Interfaces 2016, 8, 24722

[VI] Charge Transfer State Separation at the TQ1/PC71BM Interface R. Volpi, M. Linares

Submitted 


\section{Contribution to the Papers}

\section{Paper I}

I have been introduced to the project by my supervisor Mathieu Linares and my co-supervisor Sven Stafström. I played an active role in the conception of the theoretical model. I improved a previously existing code of the project to handle a realistic correlation of the enrgetic landscape through induced dipoles. I executed and analyzed all the calculations, and I wrote the majority of the manuscript.

\section{Paper II}

I proposed the polarization part of the theoretical model used during discussion with my supervisor Mathieu Linares. I completely restructured the code and made it capable of considering interactions with atomic charges and induced dipoles on nearby molecules. With the help of Mathieu Linares and my cosupervisor Sven Stafström I planned executed and analyzed all the calculations, and I wrote the majority of the manuscript.

\section{Paper III}

Leonardo Sousa a PhD student from the group of Prof. da Silva Filho from University of Brasilia came to visit our group. Together with my supervisor Mathieu Linares I participated to the conceivement of the project for this Paper. With the help of Mathieu Linares I improved the user interface of the code and I subsequently introduced Leonardo to it. I proposed the explanation of the shape of the $\mathrm{O}$ energies and of the mobility field dependencies of this system. I helped Leonardo with technical details and code developments for the analysis. I participated in the writing of the manuscript and particularly to its finalization.

\section{Paper IV}

I wrote the code to systematically cut the alkyl chains to be able to study systems of big molecules. With the help of my supervisor I obtained the force field for the three molecules under study. I achieved a realistic morphology of the systems trough MD simulations. I analyzed the population of transfer integrals for the different systems and studied their correlation with rise, twist and shift distribution. I participated to the writing of the manuscript.

\section{Paper V}

I rewrote the $\mathrm{KMC}$ procedure to allow the treatment of multiple charges and changed the stored simulation data accordingly. I was involved in the supervision 
of the master student Racine Nassau that took part in this study. With my help and the help of Mathieu Linares, Racine planned and executed all the calculations. I helped her with the analysis of the data and I formulated the CT state splitting diagram. I participated to the writing of the manuscript and particularly to its finalization.

\section{Paper VI}

My supervisor Mathieu Linares proposed the system studied in this paper and introduced me to the project. I restructured the code to handle a division between QM and MM part inside the same molecule. Now the core of a molecule can be specified and it interacts with the rest of the atoms that are treated exclusively in the MM part for the electrostatic interactions. The energetic levels will be calculated exclusively for the core and the charge carrier will be placed on this part of the molecule. The alkyl chains can be automatically cut by the program for all the molecules in the box and the interactions between alkyl chains and molecular core is properly handled in the program. In this way it is possible to perform KMC simulations also on big molecules like 2-TQ1. I performed all calculations and analyzed the results. I wrote the majority of the manuscript. 


\section{Papers}

The articles associated with this thesis have been removed for copyright reasons. For more details about these see:

http://urn.kb.se/resolve?urn=urn:nbn:se:liu:diva-133329 